\title{
Formation of Golgi-Derived Active Zone Precursor Vesicles
}

\author{
Christoph Maas, ${ }^{1}$ Viviana I. Torres, ${ }^{1}$ Wilko D. Altrock, ${ }^{2}$ Sergio Leal-Ortiz, ${ }^{1}$ Dhananjay Wagh, ${ }^{1}$ Ryan T. Terry-Lorenzo, ${ }^{1}$ \\ Anna Fejtova, ${ }^{2}$ Eckart D. Gundelfinger, ${ }^{2}$ Noam E. Ziv, ${ }^{3}$ and Craig C. Garner ${ }^{1}$ \\ ${ }^{1}$ Department of Psychiatry and Behavioral Sciences, Nancy Pritzker Laboratory, Stanford University, Palo Alto, California 94304-5485, ${ }^{2}$ Department of \\ Neurochemistry and Molecular Biology, Leibniz Institute for Neurobiology, Magdeburg D-39118, Germany, and ${ }^{3}$ Technion Faculty of Medicine and \\ Network Biology Research Laboratories, Haifa 32000, Israel
}

Vesicular trafficking of presynaptic and postsynaptic components is emerging as a general cellular mechanism for the delivery of scaffold proteins, ion channels, and receptors to nascent and mature synapses. However, the molecular mechanisms leading to the selection of cargos and their differential transport to subneuronal compartments are not well understood, in part because of the mixing of cargos at the plasma membrane and/or within endosomal compartments. In the present study, we have explored the cellular mechanisms of active zone precursor vesicle assembly at the Golgi in dissociated hippocampal neurons of Rattus norvegicus. Our studies show that Piccolo, Bassoon, and ELKS2/CAST exit the trans-Golgi network on a common vesicle that requires Piccolo and Bassoon for its proper assembly. In contrast, Munc13 and synaptic vesicle proteins use distinct sets of Golgi-derived transport vesicles, while RIM1 $\alpha$ associates with vesicular membranes in a post-Golgi compartment. Furthermore, Piccolo and Bassoon are necessary for ELKS2/CAST to leave the Golgi in association with vesicles, and a core domain of Bassoon is sufficient to facilitate formation of these vesicles. While these findings support emerging principles regarding active zone differentiation, the cellular and molecular analyses reported here also indicate that the Piccolo-Bassoon transport vesicles leaving the Golgi may undergo further changes in protein composition before arriving at synaptic sites.

\section{Introduction}

Neurotransmitter release occurs through the fusion of synaptic vesicles (SVs) with the active zone (AZ), a region of the plasma membrane spatially controlled by a protein meshwork called $\underline{\mathrm{C}} \mathrm{y}-$ toskeletal matrix assembled at the AZ (CAZ) (Sudhof, 2004). Proteins of the CAZ include members of the Munc13, RIM, Bassoon and Piccolo, and ELKS/CAST families (Schoch and Gundelfinger, 2006). At present, our understanding of the temporal and spatial control of synapse assembly remains rudimentary (Ziv and Garner, 2004; Waites et al., 2005).

Vesicular delivery of SV and CAZ proteins to nascent synapses are thought to contribute to the rapid assembly of functional presynaptic boutons (Ahmari et al., 2000; Zhai et al., 2001; Ziv and Garner, 2004). Studies on the CAZ proteins Piccolo and Bassoon revealed that they are delivered to synapses via Golgiderived $80 \mathrm{~nm}$ dense core vesicles called Piccolo-Bassoon Trans-

\footnotetext{
Received Jan. 13, 2012; revised June 12, 2012; accepted June 22, 2012.

Author contributions: C.M., V.I.T., D.W., E.D.G., N.E.Z., and C.C.G. designed research; C.M., V.I.T., W.D.A., S.L.-0., R.T.T.-L., and A.F. performed research; C.M. analyzed data; C.M., N.E.Z., and C.C.G. wrote the paper.

This work was supported by NIH Grants NS39471 and NS353862 and United States-Israel Binational Science Foundation Grant 2007425 to C.C.G. and N.E.Z. and by the DFG (Deutsche Forschungsgemeinschaft) research fellowship to C.M. and SFB779/B9 to E.D.G. We thank T. Ohtsuku for the ELKS2 CDNA, R. Edwards for the SV2 CDNA, N. Brose for the EGFP-Munc13-1 clone, T. Sudhof for the EGFP-RIM1 $\alpha$ clone, and A. El-Husseini for the ARF1-Q71I-HA clone. We further thankC. Hoogenraad for kindly providing ELKS2-specific antibodies and L. Binder for kindly providing the Tau1-specific antibody. We also thank S. Kim for reviewing this manuscript and J. Galaz and N Y. Gedde for excellent technical assistance with the biochemistry and hippocampal cultures respectively.

Correspondence should be addressed to Dr. Craig C. Garner, Department of Psychiatry and Behavioral Sciences, Nancy Pritzker Laboratory, Stanford University, 1201 Welch Road, Palo Alto, CA 94304-5485. E-mail: cgarner@stanford.edu.

V. I. Torres's present address: Center for Advanced Microscopy, CMA Bio-Bio, Universidad de Concepción, Chile. DOI:10.1523/JNEUROSCI.0195-12.2012

Copyright $\odot 2012$ the authors $\quad 0270-6474 / 12 / 3211095-14 \$ 15.00 / 0$
}

port Vesicles (PTVs) (Zhai et al., 2001; Shapira et al., 2003; Dresbach et al., 2006). Immunoprecipitation experiments from brain light membrane fractions indicated that these vesicles also carry Munc13-1, RIM1 $\alpha$, and ELKS2 (CAST1/ERC2) (Ohtsuka et al., 2002; Shapira et al., 2003). These data suggest that a small number of PTVs, each carrying a significant complement of preassembled CAZ scaffolds, might be sufficient for the formation of functional AZs (Ziv and Garner, 2004; Waites et al., 2005), a concept consistent with quantitative immunocytochemistry experiments demonstrating that Bassoon, Piccolo, and RIM content per synapses can be accounted for by the delivery of two to three transport packets (presumably PTVs) of these molecules (Shapira et al., 2003). Furthermore, imaging studies of recombinant EGFP-tagged Bassoon revealed stepwise incorporation of mobile units of Bassoon into nascent presynaptic sites (Shapira et al., 2003; Bresler et al., 2004).

Although thought to be Golgi derived, many questions regarding PTV biogenesis remain. For example, are CAZ proteins coassembled at the Golgi network or through the fusion of multiple precursor vesicles during their recycling with the plasma membrane, similar to SV maturation (Yonekawa et al., 1998; Hannah et al., 1999)? Alternatively, given the tendency of CAZ molecules to form complexes (Wang et al., 2009), does coassembly occur en route by the recruitment of molecules to PTVs from soluble pools? To explore these possibilities, we investigated whether: (1) CAZ proteins are transported into axons in association with Golgi-derived vesicular membranes or associate with such vesicles at later steps; (2) CAZ proteins are sorted into the same transport vesicle at the Golgi; or (3) Bassoon and/or Piccolo are necessary for the proper formation of their own transport 
vesicle. Our results reveal that most CAZ proteins initially associate with vesicular membrane at the level of the Golgi; however, while Piccolo, Bassoon, and ELKS share a common Golgi-derived transport vesicle (gPTV) that requires Piccolo and Bassoon for its initial assembly, Munc13 exits the soma on a distinct Golgiderived vesicle and RIM $1 \alpha$ is recruited to vesicular membranes in a post-Golgi step. These data suggest that gPTVs may undergo changes in composition before becoming a mature PTV (mPTV) ready for insertion at nascent synapses.

\section{Materials and Methods}

Antibodies. The following antibodies were used for Western blotting (WB) and/or immunocytochemistry (IC): mouse anti-Bassoon (1:5000 for WB, 1:500 for IC; Stressgen), rabbit anti-Piccolo (1:5000 for WB, 1:500 for IC; Zhai et al., 2000), rabbit anti-ELKS2 (1:1000 for WB, 1:100 for IC), rabbit anti-ELKS2 (1:1000 for WB, 1:100 for IC; gift from C. Hoogenraad, Erasmus Medical Center, Rotterdam, The Netherlands), mouse anti-Munc13-1 (1:1000 for WB, 1:100 for IC; Synaptic Systems), rabbit anti-RIM1 $\alpha$ (1:1000 for WB, 1:100 for IC; Synaptic Systems), mouse anti-RIM1 $\alpha$ (1:100 for IC, Transduction Laboratories), mouse anti-SV2 (1: 5000 for WB, 1:500 for IC, Developmental Studies Hybridoma Bank), mouse anti-Tubulin (1:5000 for WB; Sigma-Aldrich); rabbit anti-Synaptotagmin (1:1000 for WB; BD Biosciences), mouse anti-TGN38 (1:2000 for WB, 1:200 for IC; BD Biosciences), rabbit anti-ELKS1b/2 (ERC1b/2, 1:200 for IC; Synaptic Systems), mouse antiTau1 (1:500 for IC, gift from L. Binder, Northwestern University, Chicago, IL), mouse anti-PSD-95 (1:100 for IC; Affinity BioReagents), chicken anti-HA (1:500 for IC; Bethyl Laboratories), mouse anti-GM130 (1:200 for IC; Transduction Laboratories), rabbit anti-GM130 (1:200 for IC; Calbiochem), and rabbit anti-Synaptophysin (1:200 for IC; Santa Cruz Biotechnology).

Constructs. The monomeric RFP (mRFP)-tagged Bassoon (mRFPBsn, amino acids 95-3938 of Bassoon) was created by replacing EGFP with mRFP in EGFP-Bsn (Dresbach et al., 2006) using the SnaB1 and AccIII sites. Similarly, mRFP-BsnGBR (amino acids 2088-2564 of Bassoon) was constructed by replacing EGFP with mRFP in EGFP-BsnGBR (Dresbach et al., 2006) using the NheI and EcoRI sites. ELKS2 cDNA was generously provided by Toshihisa Ohtsuka (University of Yamanashi, Yamanashi, Japan; Deguchi-Tawarada et al., 2004). As N- and C-terminal tagging of ELKS2 lead to aggregation or mislocalization of ELKS2 in neurons (data not shown), EGFP was inserted internally into a low complexity region (predicted by SMART; Schultz et al., 1998) lying outside of any known protein domains. This was accomplished by initially subcloning an ELKS2 PCR product into the AscI and BstEII sites of the pCR-Blunt vector (Invitrogen). A linker containing an NheI and a SacII site was then inserted at the AgeI site (bp 2795) of ELKS2 and EGFP subcloned as an NheI-SacII fragment into ELKS2. Finally, ELKS2 with the internal EGFP (iEGFP) was subcloned as an AscI-BstEII fragment into the FUGW vector (Lois et al., 2002). This new ELKS2-iEGFP now nicely localizes to synapses without forming intracellular aggregates. EGFP-Munc13-1 was generously provided by Nils Brose (Max Planck Institute for Experimental Medicine, Göttingen, Germany; Kalla et al., 2006) and EGFP-RIM1 $\alpha$ by Thomas Südhof (Stanford University, Stanford, CA), with the EGFP cloned into the BspEII and NheI sites between the zinc finger and the PDZ domain of RIM1 $\alpha$. SV2 cDNA was a generous gift from Robert Edwards (University of California at San Francisco, San Francisco, CA). EGFP-SV2 was created by cloning an SV2 PCR product into the BspEI and EcoRI sites of EGFP-C1. ARF1-Q71I-HA was a generous gift from Alaa El-Husseini (University of British Columbia, Vancouver, Canada). pZoff vectors expressing shRNAs (Pclo28 or Bsn16) against Piccolo and Bassoon, respectively, were used as described previously (Leal-Ortiz et al., 2008).

A Piccolo/Bassoon double knockdown (DKD) vector and the DKDscrambled control vector (scDKD) were created by cloning Pclo28 and Bsn16 or their scrambled controls (Leal-Ortiz et al., 2008) in tandem within the pZoff-vector with a 750bp spacer between the H1-Pclo28 and U6-Bsn16 transcription units. In brief, a 750 bp spacer PCR product containing EcoRI and BstBI sites at the $3^{\prime}$-end was subcloned as a BsiWI-
AccIII fragment into the BsiWI and BstBI sites of FUGWTS28 (LealOrtiz et al., 2008). The BstWI site was disrupted during this cloning. Bsn16 was subcloned as an AccIII-EcoRI fragment into the BstB1 and EcoRI sites of the spacer. The control vector (scDKD) was cloned accordingly. The Bsn 16 resistant version of full-length mRFP-tagged Bassoon $\left(\mathrm{mRFP} \mathrm{Bsn}^{*}\right)$ was created with site-directed mutagenesis using the mRFP-Bsn construct with the following oligonucleotides (forward primer: 5' -CACATCAACCTCCAGCCAGCCAAATTTCAATACCTGTACCCAGTGCCACAACAAGG; reverse primer: 5'-GGTTGAACCCACATTGG TTG CAGACCTTGTTGTGGCACTGGGTACAGGTATTGAAATTTGGC).

Lysate fractionation. All steps were conducted either on ice or at $4^{\circ} \mathrm{C}$. Rat brains at indicated ages were homogenized in buffer A [5 mM MES (2-(N-morpholino)ethanesulfonic acid), pH 7.0, $0.3 \mathrm{~m}$ sucrose, $1 \mathrm{~mm}$ EDTA) containing Protease Inhibitor Cocktail (Roche Biochemicals) with 10 strokes in a Teflon glass homogenizer. The homogenate was centrifuged at $1000 \times g$ for $15 \mathrm{~min}$. Post-Nuclear Supernatant (PNS) was hypotonically lysed with 10 vol of 5 mM MES, pH 7.0, plus 1 mM EDTA and incubated for $30 \mathrm{~min}$ at $4^{\circ} \mathrm{C}$ with gentle stirring. Then, the lysed PNS was centrifuged at $100,000 \times g$ for $1 \mathrm{~h}$. This generated a supernatant $\left(\mathrm{S}_{100}\right)$ and a pellet $\left(\mathrm{P}_{100}\right)$ fraction. The $\mathrm{P}_{100}$ was resuspended in buffer $\mathrm{A}$ and loaded on top of a discontinuous sucrose gradient of $0.8,1.2$, and 2.0 M. The gradient was centrifuged for $3 \mathrm{~h}$ at $270,000 \times g$ in a SW28 or SW41Ti ultracentrifuge rotor (Beckman Coulter). This generated peak 1 $(0.3 / 0.8 \mathrm{M}$ interface), peak $2(0.8 / 1.2 \mathrm{M}$ interface $)$, and peak $3(1.2 \mathrm{M} / 2.0 \mathrm{M}$ interface).

For Western blotting, protein samples were separated by SDS-PAGE using 3-8\% gradient Tris-acetate gels (Invitrogen), transferred to nitrocellulose membranes (GE Healthcare), incubated in blocking solution (5\% nonfat dry milk, 0.05\% NP-40, 150 mu NaCl, 50 mm Tris, $\mathrm{pH} 7.5$ ), and probed with primary and secondary horseradish peroxidase (HRP)conjugated antibodies (GE Healthcare) in blocking solution. Protein bands were visualized by HRP chemiluminescence (PerkinElmer) and exposure to film.

Yeast two-hybrid experiments. For cDNA library screening, the Matchmaker Two-Hybrid System 2 (Clontech Laboratories) was used with a rat brain Matchmaker cDNA library (Clontech Laboratories) as prey and Bassoon fragment RB26 (BsnGBR) as bait. Transformation and selection were performed according to the manufacturer's protocols. For narrowing down the Bassoon oligomerization domain, the Matchmaker System 3 (Clontech Laboratories) was used. In detail, the Bassoon constructs based on pGBKT7 were cotransformed with RB26 or RB28 (BsnCC3) in pGADT7 vectors into AH109 yeast cells using standard transformation protocols. Cotransformed cells were selected by growth on Leu- and Trp-lacking medium. The interaction of coexpressed proteins activating expression of reporter genes was monitored as growth on selection media lacking adenine, His, Leu, and Trp and supplemented with $1 \mathrm{~mm}$ 3-amino-1,2,4-triazole after 4 and $7 \mathrm{~d}$. Potential self-activation of constructs was always tested in parallel by cotransformation with empty prey or bait vectors.

COS7 cell cultures and transfection. COS7 cells were grown in DMEM plus $10 \%$ fetal bovine serum and penicillin/streptomycin on $165 / \mathrm{mm}^{2}$ plastic coverslips. Transfections were conducted on confluent (90$100 \%$ ) cell cultures with Lipofectamine 2000 (Invitrogen) using $4 \mu \mathrm{g}$ of total DNA and $10 \mu \mathrm{l}$ of Lipofectamine 2000 per coverslip.

Lentivirus production. Lentivirus was produced by transfecting a threeplasmid vector system comprising a shuttle plasmid (FUGW) and two packaging plasmids (pCMV R8.9 and pHCMV VSVg) into HEK293T cells grown in DMEM plus 10\% fetal bovine serum and penicillin/streptomycin as described previously (Lois et al., 2002). In brief, transfections were conducted on confluent $(90-100 \%)$ cell cultures with Lipofectamine 2000 (Invitrogen) using $22.5 \mu \mathrm{g}$ of total DNA and $60 \mu \mathrm{l}$ of Lipofectamine 2000 per $10 \mathrm{~cm}$ plate. Two days after transfection, the virus-containing medium was collected, passed through a $0.45 \mu \mathrm{m}$ filter to remove cell debris, and frozen at $-80^{\circ} \mathrm{C}$. The viral titer was determined by fluorescence analysis of infected HEK293T cells.

Hippocampal cultures. Hippocampal cultures were prepared using a modified Banker culture protocol (Banker and Goslin, 1998). In brief, hippocampi from embryonic (embryonic day 18 or 19) Sprague Dawley rats of either sex were dissected out and dissociated in $0.05 \%$ TrypLE 
(Invitrogen). Cells were plated at a density of $165 / \mathrm{mm}^{2}$ on poly-L-lysinecoated coverslips (Carolina Biological Supply). One hour after plating, coverslips were transferred in pairs to $60 \mathrm{~mm}$ dishes containing a glial feeder layer where they were inverted and maintained in Neurobasal medium containing B27 and GlutaMAX (all from Invitrogen).

Hippocampal transfection and lentivirus infection. Hippocampal neurons were transfected or infected with the plasmids or lentivirus, respectively, at the time of plating (0 DIV). The plasmids were introduced by electroporation of cells in suspension, conducted in $0.4 \mathrm{~cm}$ Gene Pulser Cuvettes (Bio-Rad Laboratories) using $4 \times 10^{6}$ cells in $500 \mu \mathrm{l}$ of gliaconditioned medium (10\% FBS and $20 \mathrm{~mm}$ glucose in glutamine-free MEM) and $20 \mu \mathrm{g}$ of plasmid with a Gene Pulser II electroporator (BioRad Laboratories) at $0.25 \mathrm{kV}$ and $975 \mu \mathrm{F}$. Lentiviral infection was conducted on the day of plating after coverslips were transferred into $60 \mathrm{~mm}$ dishes using $10 \mu$ l of virus per dish to infect $20-30 \%$ of cells (for imaging experiments) and $100 \mu \mathrm{l} /$ dish to infect $\sim 100 \%$ of cells. Hippocampal neurons were transfected at later time points using a calcium phosphate coprecipitation protocol (Jeyifous et al., 2009). In brief, for each coverslip $2 \mu \mathrm{g}$ of DNA and $7.5 \mu \mathrm{l}$ of $2 \mathrm{M} \mathrm{CaCl}_{2}$ in $60 \mu \mathrm{l}$ volume was added dropwise to $60 \mu \mathrm{l} 2 \times \mathrm{HBS}\left(274 \mathrm{~mm} \mathrm{NaCl}, 10 \mathrm{~mm} \mathrm{KCl}, 1.4 \mathrm{~mm} \mathrm{Na}_{2} \mathrm{HPO}_{4}, 15 \mathrm{~mm}\right.$ glucose, $42 \mathrm{~mm}$ HEPES, pH 7.1), incubated for $20 \mathrm{~min}$, and then added to cultured neurons in $1 \mathrm{ml}$ of conditioned medium containing $10 \mu \mathrm{M}$ $\mathrm{CNQX}$ and $50 \mu \mathrm{M} \mathrm{APV}$. After a $30 \mathrm{~min}$ of incubation at $37^{\circ} \mathrm{C}$, neurons were rinsed $3 \times$ with $2 \mathrm{ml}$ of prewarmed HBSS and transferred back into culture dishes.

Immunocytochemistry. Hippocampal neuronal cultures, grown for 5-16 DIV, were fixed with either $4 \%$ formaldehyde and $4 \%$ sucrose in $1 \times$ PBS for $12 \mathrm{~min}$ at room temperature or $100 \%$ ice-cold methanol for $15 \mathrm{~min}$ at $-20^{\circ} \mathrm{C}$. Cells were then permeabilized with $0.25 \%$ Triton $\mathrm{X}-100$ in $1 \times$ PBS for $2 \mathrm{~min}$, washed in PBS, incubated in blocking solution ( $2 \%$ bovine serum albumin, $2 \%$ glycine, and $0.2 \%$ gelatin in 50 $\mathrm{mM} \mathrm{NH} \mathrm{N}_{4} \mathrm{Cl}$ ) for $30 \mathrm{~min}$ at room temperature, and incubated for $1 \mathrm{~h}$ at room temperature with primary antibodies in blocking solution. Afterward, cells were washed three to four times in PBS for $2 \mathrm{~min}$, incubated for $1 \mathrm{~h}$ at room temperature with secondary antibodies in blocking solution, washed again three to four times in PBS for 2 min followed by a final wash in deionized water for $2 \mathrm{~min}$, dried, and mounted in Vectashield mounting solution (Vector Laboratories).

Fluorescence images were acquired using MetaMorph 7.6 (Universal Imaging) and a Yokogawa CSU 10 spinning disc confocal head (PerkinElmer) fitted on a Zeiss Axiovert 200M microscope equipped with a Plan-Apochromat $63 \times 1.4$ numerical aperture $(N A)$ and a F-Fluar $40 \times 1.3 \mathrm{NA}$ objective. Images were processed using ImageJ (National Institutes of Health) and Adobe Photoshop software.

Time-lapse imaging. For the time-lapse imaging experiments, culture medium was replaced with Tyrode's buffer $(25 \mathrm{~mm}$ HEPES, $119 \mathrm{~mm}$ $\mathrm{NaCl}, 2.5 \mathrm{~mm} \mathrm{KCl}, 30 \mathrm{~mm}$ glucose, $2 \mathrm{~mm} \mathrm{CaCl}$, $2 \mathrm{~mm} \mathrm{MgCl}_{2}, \mathrm{pH}$ 7.3). Cultured neurons were maintained at $37^{\circ} \mathrm{C}$ by placing coverslips in a custom-designed environmental chamber affixed to the microscope stage. Sequential images were acquired at $1 \mathrm{~s}$ exposures every 15-30 s using MetaMorph 7.6 (Universal Imaging) and a Yokogawa CSU 10 spinning disc confocal head (PerkinElmer) fitted on a Zeiss Axiovert $200 \mathrm{M}$ microscope, and a F-Fluar $40 \times$ NA1.3 objective. Images were processed using ImageJ (National Institutes of Health) and Adobe Photoshop software.

Image analysis. Quantification of fluorescence data was performed using MetaMorph (Universal Imaging) and ImageJ software (National Institutes of Health). Somatic Golgi localization was assayed by setting the $z$ plane limits for acquisition $(0.50-1.0 \mu \mathrm{m}$ per slice, $5-10$ slices $)$ on the basis of the fluorescence signal for the Golgi markers (GM130 or TGN38). The Golgi marker signal was then thresholded and used to create a binary mask. Signal intensity of pixels inside the Golgi mask was measured for the costained protein in each plane. Averaged pixel intensities for the entire $z$ stack were calculated. The non-Golgi signal intensity of the costained protein was measured as average pixel intensity in a small box drawn outside of the Golgi but inside of the soma, where fluorescence exhibited an even distribution. Background signal intensity measured as average pixel intensity in a small box drawn outside of the cell was subtracted from both values. A Golgi-to-soma (non-Golgi) ratio was calculated for each cell. Experiments were conducted from a minimum of two independent culture preparations, with 10 neurons per experimental group. Colocalization of synaptic proteins in initial axonal segments was estimated using the ImageJ colocalization plugin.

Statistics. Statistical comparisons for all confocal analyses were made using two-tailed Student's $t$ tests using Microsoft Excel (Microsoft).

\section{Results}

\section{Munc13-1 and ELKS but not RIM1 $\alpha$ associate with Golgi membranes}

Our initial studies of Bassoon revealed that it first becomes associated with membranes within the trans-Golgi Network (TGN) that bud forming an axonally transported vesicular intermediate (Dresbach et al., 2006). To explore whether ELKS2, Munc13-1, and RIM $1 \alpha$ also become associated with vesicular membranes at the Golgi, we used two different manipulations to disrupt protein export from the Golgi. Specifically, we inhibited vesicle budding from the Golgi in hippocampal neurons by incubating cultures at $19^{\circ} \mathrm{C}$ for $2 \mathrm{~h}$ (Fig. $1 A-D$, second rows) or by expression of a constitutively active Arf1 mutant, Arf1-Q71I (Dascher and Balch, 1994; Jeyifous et al., 2009) (Fig. $1 A-D$, fourth rows). In these studies, neurons 9 DIV were coimmunostained for endogenous Bassoon (Fig. 1A), ELKS1b/2 (Fig. 1B), Munc13 (Fig. 1C), and RIM1 $\alpha$ (Fig. 1D) and markers of the cis- or trans-Golgi network (GM130 or TGN38) and imaged by confocal microscopy. Our experiments with Bassoon (Fig. 1A) confirmed the previous reported association with the Golgi (Dresbach et al., 2006). Similarly, inhibition of Golgi export leads to the accumulation of ELKS1b/2 at the TGN in cultured hippocampal neurons following a $19^{\circ} \mathrm{C}$ temperature block or Arf1-Q71I overexpression (Fig. $1 B)$. Interestingly, Munc13-1 accumulates at the cis-Golgi under these conditions, colocalizing with cis-Golgi marker GM130 (Fig. $1 C$ ) but not TGN38 (data not shown). In contrast, RIM1 $\alpha$ was not observed to accumulate at any part of the Golgi (Fig. 1D).

To quantify the effects of Golgi export inhibition, we converted the Golgi marker signal into a binary mask and assessed the signal intensity of each protein of interest inside the mask in comparison to a corresponding region of the cell soma outside the Golgi mask (Fig. 1E). This comparison is given as a ratio (Golgi/non-Golgi) in which a value of 1.0 indicates no particular Golgi association, as there is no difference between the signals inside and outside of the mask. A Z-projection from three confocal planes was used for each cell analyzed. Quantification confirms both significant increase of ELKS1b/2 and Munc13 at the Golgi after $19^{\circ} \mathrm{C}$ temperature block (Fig. 1 E, black bars) or Arf1Q71I (Fig. 1E, gray bars) overexpression as well as no effect on RIM $1 \alpha$ under either of these conditions. The control bars (Fig. $1 E$, white bars) show quantification at $37^{\circ} \mathrm{C}$ (Fig. $1 A-D$, first rows), which exhibits no significant difference to the control cells expressing mRFP (Fig. $1 A-D$, third row, quantification not shown). We also performed these experiments with Piccolo, showing similar results as Bassoon (Fig. $1 E$ ). The transmembrane synaptic vesicle protein SV2 and microtubule-associated protein Tau were used as positive and negative controls, respectively (Fig. $1 E$ ).

To verify the findings for RIM $1 \alpha$ and ELKS2, we conducted a number of additional experiments. First, we used a second antibody to detect RIM $1 \alpha$ at the Golgi and fixed neurons with methanol to remove soluble RIM $1 \alpha$ after $19^{\circ} \mathrm{C}$ incubation. Again, we failed to detect an accumulation of $\operatorname{RIM} 1 \alpha$ at the Golgi after inhibiting transport vesicle formation (Fig. $1 F$ ). Second, we examined whether temperature block cause Golgi accumulation of EGFP-RIM $1 \alpha$. Once more, we failed to detect an accumulation of 
A

$37^{\circ} \mathrm{C}$

$19^{\circ} \mathrm{C}$
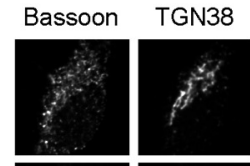

merge
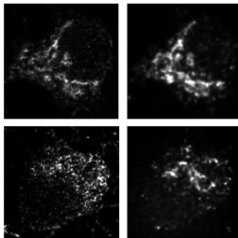

mRFP

Arf1-Q711
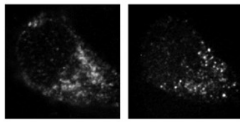

C

$37^{\circ} \mathrm{C}$

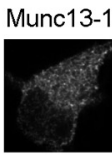

GM130
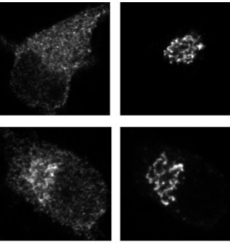

$19^{\circ} \mathrm{C}$

mRFP
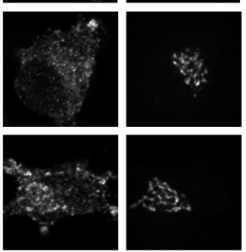
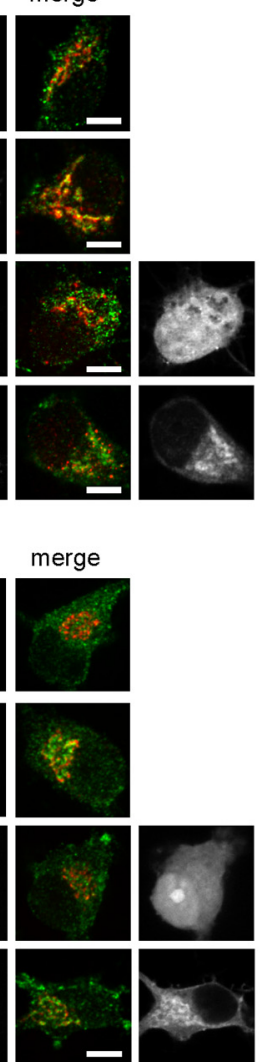

merge
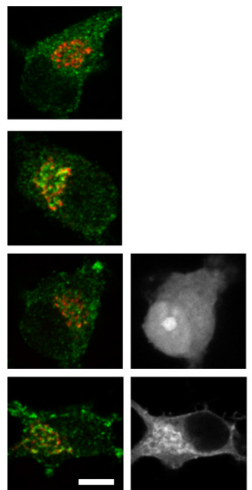

B

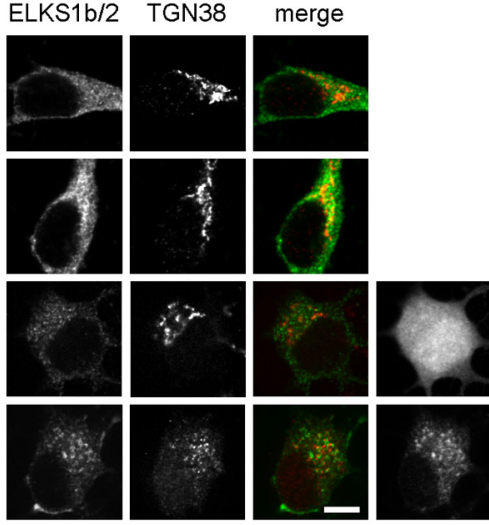

D
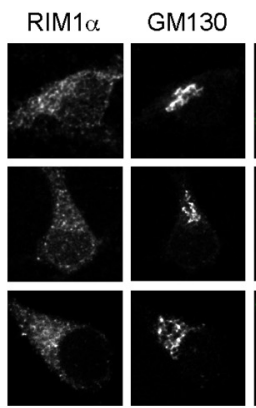

\section{merge}
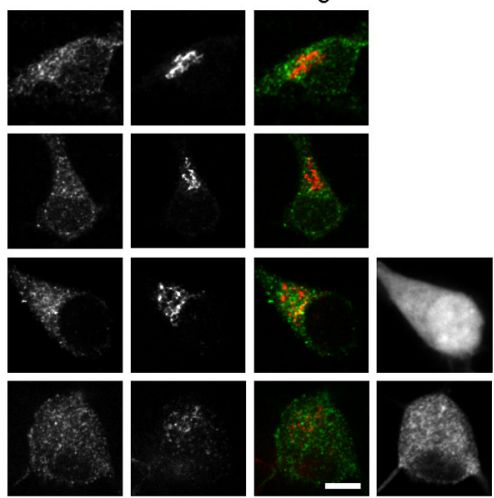

E

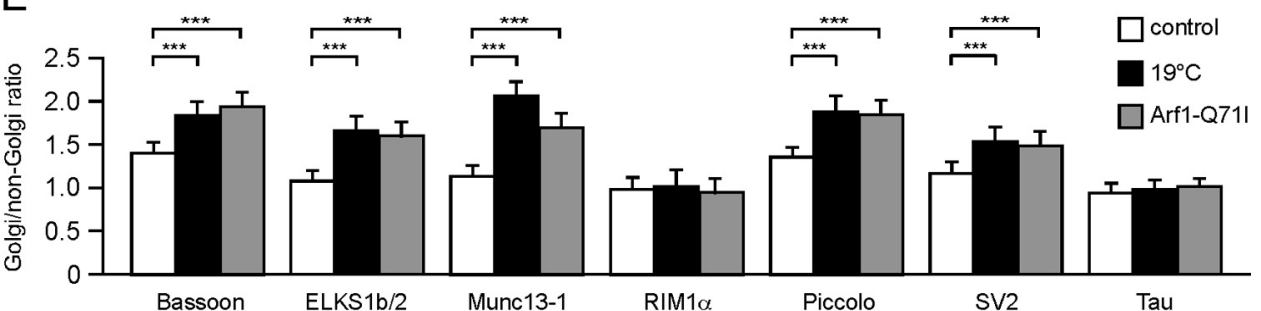

F

RIM1 $\alpha$ (MeOH/ SynSys)
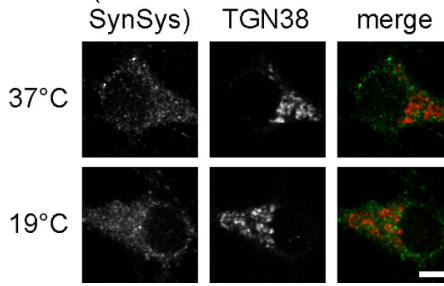

G

EGFP-

RIM1 $\alpha$
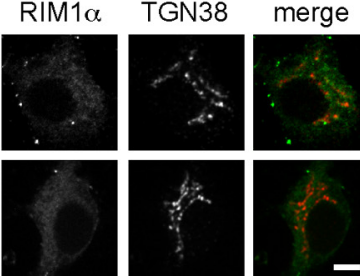

$\mathrm{H}$

ELKS2-

iEGFP TGN38 merge
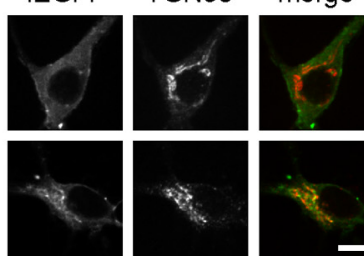

Figure 1. Association of Basoon, ELKS1b/2, Munc13-1, RIM1 $\alpha$, and Piccolo with Golgi membranes. $\boldsymbol{A}-\boldsymbol{D}$, Confocal images of cell soma of hippocampal neurons 9 DIV immunostained with antibodies against the Golgi markers TGN38 $(\boldsymbol{A}, \boldsymbol{B})$ or GM130 $(\boldsymbol{C}, \boldsymbol{D})$, as well as Bassoon $(\boldsymbol{A})$, ELKS1b/2 $(\boldsymbol{B})$, Munc13-1 $(\boldsymbol{C})$, or RIM1 $\alpha(\boldsymbol{D})$ following a $2 \mathrm{~h}$ incubation at $19^{\circ} \boldsymbol{C}(\boldsymbol{A}-\boldsymbol{D}$, second row) or after expressing the constitutively active Arf1 mutant Arf- $0711-\mathrm{HA}$ for $14 \mathrm{~h}\left(\boldsymbol{A}-\boldsymbol{D}\right.$, fourth row). Neurons incubated at $37^{\circ}(\boldsymbol{A}-\boldsymbol{D}$, first row) or expressing mRFP for $14 \mathrm{~h}(\boldsymbol{A}-\boldsymbol{D}$, third row) serve as controls. Scale bars $(\boldsymbol{A}-\boldsymbol{D}), 5 \mu \mathrm{m}$. $\boldsymbol{E}$, Quantification of $\boldsymbol{A}-\boldsymbol{D}$ and respective experiments immunostained with antibodies against the synaptic vesicle protein $S V 2$, the microtubule-associated protein Tau, and the CAZ protein Piccolo. Data are represented as mean $\pm S D,{ }^{* * *} p<0.005, N=30$ cells from three separate experiments for each quantification. $\boldsymbol{F}$-H, Cell soma of hippocampal neurons 9 DIV immunostained with antibodies against the Golgi marker TGN38 as well as an antibodies against RIM1 $\alpha(\boldsymbol{F})$ or expressing EGFP tagged fusion proteins of RIM1 $\alpha(\boldsymbol{G})$ or ELKS2 $(\boldsymbol{H})$ following a $2 \mathrm{~h}$ incubation at $19^{\circ} \mathrm{C}\left(\boldsymbol{F}-\boldsymbol{H}\right.$, second row). Neurons incubated at $37^{\circ} \mathrm{C}(\boldsymbol{F}-\boldsymbol{H}$, first row) served as controls. Neurons in $\boldsymbol{E}$ were fixed using methanol to remove soluble proteins from the cells. Scale bars $(\boldsymbol{F}-\boldsymbol{H}), 5 \mu \mathrm{m}$.

RIM1 $\alpha$ at the Golgi (Fig. $1 G$ ). As the previously used antibody detects both ELKS family members (ELKS1 and 2), we performed a $19^{\circ} \mathrm{C}$-temperature block experiment with neurons expressing EGFP-tagged ELKS2 (ELKS2-iEGFP), which is primarily expressed in the brain (Ohtsuka et al., 2002). As observed for en- dogenous ELKS1b/2, ELKS2-iEGFP accumulated at the Golgi at $19^{\circ} \mathrm{C}($ Fig. $1 \mathrm{H})$. Taken together, these results indicate that in addition to Bassoon, the CAZ proteins Munc13-1, ELKS2, and Piccolo all begin their journey to synapses by associating with Golgi-derived transport vesicles. However, the lack of RIM $1 \alpha$ at 
A Ultracentrifugation

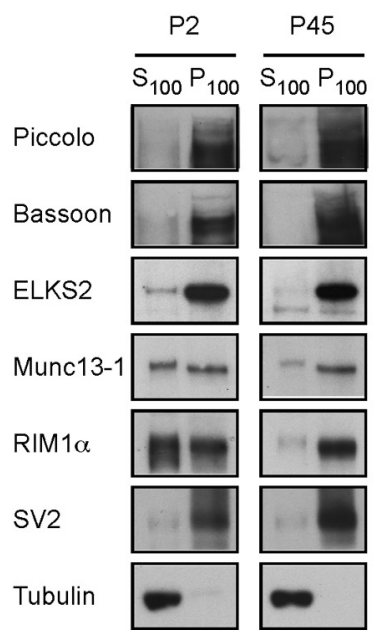

$B$ Sucrose density gradient fractionation of $P_{100}$

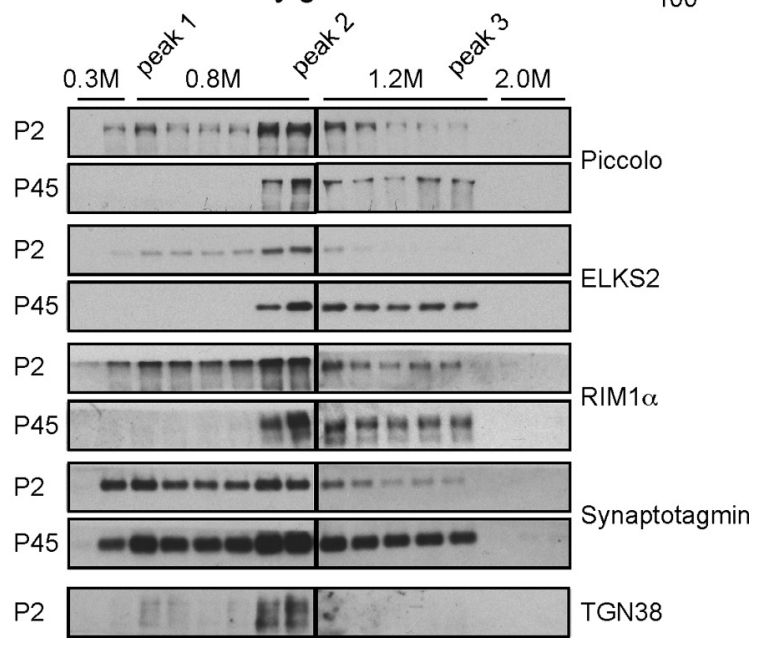

Figure 2. Active zone proteins are associated with vesicular membranes. $A$, Membrane-enriched $\left(\mathrm{P}_{100}\right)$ and soluble protein $\left(S_{100}\right)$ fractions after $100,000 \times g$ ultracentrifugation of $\mathrm{P} 2$ and $\mathrm{P} 45$ rat brains were analyzed by Western blotting for the distribution of Piccolo, Bassoon, ELKS2, Munc13-1, RIM1 $\alpha$, and the synaptic vesicle marker SV2. The cytoskeletal protein Tubulin served as a control. $\boldsymbol{B}$, Sucrose density gradient fractionation of $\mathrm{P}_{100}$ fractions from $\boldsymbol{A}$ was analyzed by Western blotting for the distribution of Piccolo, ELKS2, RIM1 $\alpha$, Synaptotagmin, and the Golgi marker TGN38.

the Golgi indicates that it probably becomes associated with vesicular membranes in a post-Golgi compartment, e.g., axonal growth cones (Shapira et al., 2003).

\section{RIM1 $\alpha$ associates with Golgi membranes in a post-Golgi compartment}

To confirm that RIM $1 \alpha$ associates with vesicular membranes, we biochemically fractionated lysates from postnatal day 2 (P2) and day $45(\mathrm{P} 45)$ rat brain by $100,000 \times$ g ultracentrifugation and discontinuous sucrose density gradient centrifugation. As reported previously, after ultracentrifugation Bassoon, Piccolo, and the transmembrane SV marker SV2 were mainly present in the membrane-enriched $\mathrm{P}_{100}$ fraction, whereas the cytosolic protein Tubulin remained in the soluble $S_{100}$ fraction (Fig. 2A) (Cases-Langhoff et al., 1996; Zhai et al., 2001). The CAZ proteins ELKS2, Munc13-1, and RIM1 $\alpha$ were detected in both $S_{100}$ and $\mathrm{P}_{100}$ fractions in P2 lysates. Similar to Bassoon and Piccolo, a majority of ELKS2 is found in the $\mathrm{P}_{100}$ fraction at this time. In contrast, Munc13-1 and RIM1 $\alpha$ partitioned fairly equally between $\mathrm{S}_{100}$ and $\mathrm{P}_{100}$ fractions in P2 lysates and were predominantly found in the $\mathrm{P}_{100}$ fraction in adult $\mathrm{P} 45$ lysates (Fig. $2 \mathrm{~A}$ ). To further characterize the membrane-associated proteins, $\mathrm{P}_{100}$ fractions from both P2 and P45 brains were separated by ultracentrifugation with discontinuous sucrose density gradients. Here, Piccolo, ELKS2, and RIM1 $\alpha$ exhibited a similar pattern, appearing in peaks around the $0.3 / 0.8 \mathrm{M}$ (Peak 1), the $0.8 / 1.2 \mathrm{M}$ (Peak 2), and the 1.2/2.0 M (Peak 3) interfaces in P2 and P45 rat brain (Fig. 2 B). Peak 1 contains light membrane fractions and a variety of vesicular transport vesicles, including SVs (Huttner et al., 1983) and PTVs (Zhai et al., 2001) and growth cone proteins (Pfenninger et al., 1983; Lohse et al., 1996). Peak 2 comprises mostly microsomal/Golgi membranes (Cotman and Matthews, 1971) containing, among others, the trans-Golgi network marker TGN38 (Fig. 2B), while Peak 3 is thought to contain synaptosomal membranes that accumulate CAZ as well as PSD proteins during development (Cotman and Matthews, 1971; Langnaese et al., 1996; Zhai et al., 2001). Interestingly, CAZ proteins in light membranes of peak 1 decrease during development (Fig. $2 B$ ), reflecting a presumed reduction in vesicular transport intermediates in older neurons due to reduced synaptogenesis at this stage. However, a reduction of the SV protein Synaptotagmin was not observed in P45 light membrane fractions (Fig. 2B), which is consistent with the continued biogenesis and maintenance of SV pools both during development and in the mature brain.

These results indicate that $\operatorname{RIM} 1 \alpha$ is indeed associated with vesicular membranes. However, as we failed to detect RIM1 $\alpha$ at Golgi membranes, we conclude that its initial membrane association must occur in a post-Golgi compartment. At present, it is unclear whether this takes place while vesicles transit down the axon or during a recycling step with the plasma membrane. Based on the punctate pattern and colocalization of RIM1 $\alpha$ with other CAZ proteins in axonal growth cones as well as its quantal recruitment into synapses similar to Piccolo and Bassoon (Shapira et al., 2003), we speculate that RIM1 $\alpha$ may be recruited to vesicular membrane from soluble pools within axons or growth cones, perhaps via its association with ELKS (Ohtsuka et al., 2002).

\section{Bassoon contains an oligomerization domain}

Having demonstrated that the CAZ proteins Piccolo, Bassoon, ELKS2, and Munc13-1 associate with Golgi membranes at the cell soma, we examined whether these CAZ proteins are trafficked into the axons via a common Golgi-derived transport vesicle. Previous studies did not examine when or where complex formation between CAZ molecules occurred, but only demonstrated that they existed in complexes within light membranes and colocalized in distal axonal segments and growth cones (Zhai et al., 2001; Shapira et al., 2003). To resolve this issue, we took advantage of a dominant-negative Bassoon construct (Bassoon Golgi Binding Region or BsnGBR) containing the coiled-coil 2 (CC2) region of Bassoon and previously shown to accumulate on Golgi membranes and reduce the synaptic levels of endogenous Bassoon (Dresbach et al., 2006). At first, we performed a series of experiments aimed at understanding how BsnGBR acts at the Golgi to disrupt the forward trafficking of Bassoon to synapses.

In our first experiments, we performed a yeast two-hybrid screen with a BsnGBR domain construct (RB26; aa 2088-2563; Fig. 3A). This region of Bassoon selected cDNA clones spanning the homologous CC2 sequences from Bassoon (e.g., clone RB29; aa 1692-3263; Fig. 3A) and Piccolo (data not shown) and suggested that sequences within BsnGBR contain a hetero-/homooligomerization domain (data not shown). Further mapping revealed that BsnGBR interacted with two regions within RB29, one that comprised almost exclusively the CC2 region of Bassoon (RB38; aa 2349-2469) as well an ill-defined region just amino terminal to CC2 (RB36; aa 1805-2237) (Fig. 3A). BsnGBR (RB26) did not interact with CC3 (Fig. 3A).

To confirm a role for the CC2 region as a homo-interacting domain, we used Lipofectamine 2000 to transfect and coexpress in COS-7 cells mRFP-tagged BsnGBR (mRFP-BsnGBR) with either EGFP-tagged full-length Bassoon, the central core domain of Bassoon (BsnCD, amino acids 1692-3263) containing the CC2 

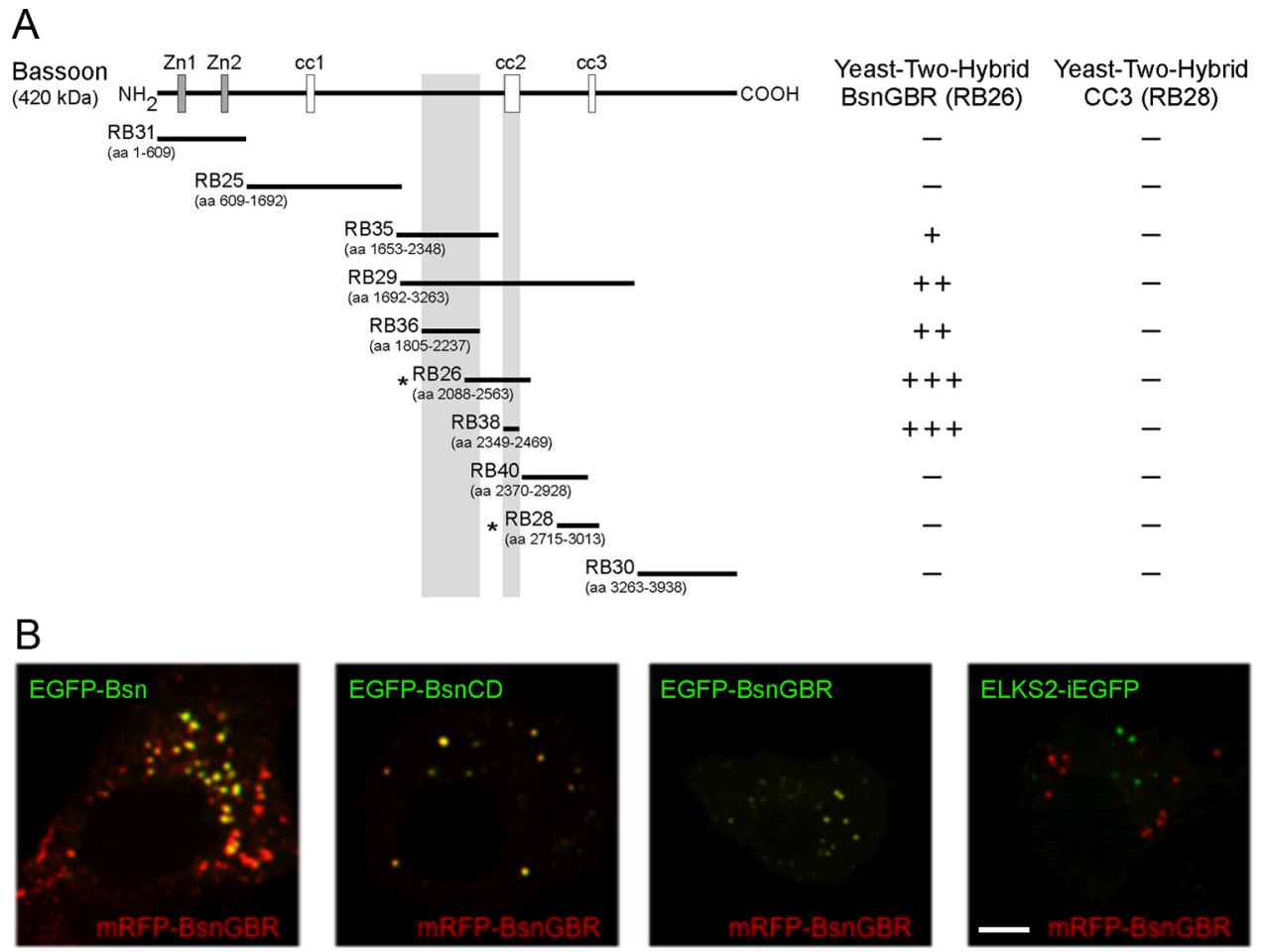

Figure 3. The Bassoon Golgi binding region contains an oligomerization domain. $A$, Yeast two-hybrid experiments with different segments of Bassoon testing binding with BsnGBR (RB26) and the CC3 region of Bassoon (RB28). Gray bars indicate possible interaction regions. B, COS-7 cells coexpressing mRFP-BsnGBR and EGFP-tagged Bassoon, BsnCD, BsnGBR, or ELKS2, respectively. Scale bar, $5 \mu \mathrm{m}$.

and CC3 domains, or BsnGBR (Fig. 3B). All three EGFP-tagged proteins formed intracellular clusters that colocalized with coexpressed mRFP-BsnGBR. As a control, mRFP-BsnGBR was coexpressed with ELKS2-iEGFP in COS-7 cells. ELKS2-iEGFP also forms intracellular clusters, but these do not colocalize with mRFP-BsnGBR clusters. Note that mRFP-BsnGBR does not associate with Golgi membranes in COS-7 cells.

\section{ELKS2, but not Munc13-1, traffics into the axon in association with gPTVs}

To explore whether BsnGBR physically impairs the recruitment and/or export of Piccolo and Bassoon from the Golgi via gPTVs (Golgi-derived PTVs), calcium phosphate transfection was used to overexpress mRFP-BsnGBR in neurons starting at 7 DIV or 8 DIV. The subcellular distribution of endogenous Piccolo and Bassoon was then examined $1 \mathrm{~d}$ later. Bassoon, as well as Piccolo, was found to accumulate on Golgi membranes in cells overexpressing mRFP-BsnGBR (Fig. 4A,D). These data indicate that BsnGBR acts as dominant negative by preventing the forward trafficking of Bassoon and Piccolo from the Golgi, perhaps by affecting their oligomerization state, rather than displacing their association with Golgi membranes as suggested previously (Dresbach et al., 2006). Note that the monoclonal antibody used in this experiment is raised against an epitope on Bassoon not present in BsnGBR (tom Dieck et al., 1998) and does not detect mRFP-BsnGBR (data not shown). Importantly, mRFP-BsnGBR overexpression did not cause the accumulation of the SV protein SV2 on Golgi membranes (Fig. 4D), indicating that mRFPBsnGBR specifically disrupts the forward trafficking of Bassoon and Piccolo and does not generally poison membrane trafficking through the Golgi as occurs by overexpressing Arf1-Q71I. The retention of both Piccolo and Bassoon at the TGN under these conditions are consistent with earlier studies (Zhai et al., 2001;
Shapira et al., 2003; Dresbach et al., 2006; Fejtova et al., 2009), suggesting that Piccolo and Bassoon are coassembled onto the same Golgi-derived transport vesicle.

Previous studies have shown that ELKS2 directly binds the CC3 region of Piccolo and Bassoon (Deguchi-Tawarada et al., 2004; Jose et al., 2008; Ohtsuka et al., 2002) and that Munc13-1 forms a complex with Piccolo, Bassoon, ELKS2, and RIM1 $\alpha$ (Wang et al., 2009). We therefore examined whether Munc13-1 and/or ELKS utilizes gPTVs for their forward trafficking out of the Golgi and into axons by assessing their distribution in neurons overexpressing mRFP-BsnGBR. Similar to endogenous Piccolo and Bassoon, ELKS1b/2 accumulates at the Golgi in mRFP-BsnGBR-overexpressing cells, while Munc13-1 did not (Fig. 4B-D). Consistently, the overexpression of Arf1-Q71I or mRFP-BsnGBR blocked the axonal targeting of de novo synthesized EGFP-Bassoon and ELKS2-iEGFP (Fig. 4, E and F, respectively). In contrast, axonal targeting of EGFP-SV2 (Fig. 4G) or EGFP-Munc13-1 (Fig. 4H) is blocked only by overexpressing Arf1-Q71I and not by mRFP-BsnGBR. These results indicate that ELKS is coassembled with Piccolo and Bassoon at the level of the Golgi onto gPTVs, while Munc13-1 utilizes a distinct Golgiderived transport vesicle.

To verify these results, we first compared the degree of colocalization of different CAZ proteins with Bassoon in proximal axons of immature DIV5 neurons, a time when most synaptic proteins are engaged in vesicular transport (Maas et al., 2006; Zhai et al., 2000). Double-labeled immunofluorescence imaging of proximal axons revealed that endogenous ELKS1b/2, Munc13-1, Piccolo, and the SV protein Synaptophysin display some degree of colocalization with Bassoon (Fig. 5A-D). However, while Piccolo and ELKS1b/2 colocalize with Bassoon to a similar high degree of $70.5 \pm 5.7 \%$ and $67.4 \pm 8.3 \%$, respectively, the degree of colocalization of Munc13-1 or Synaptophysin with 
A

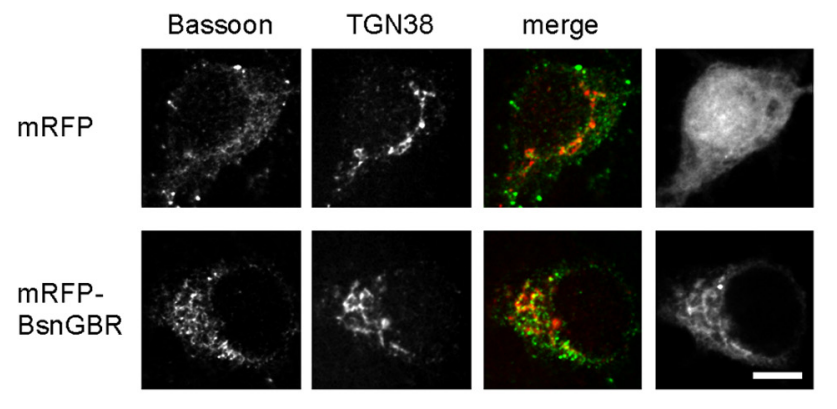

C

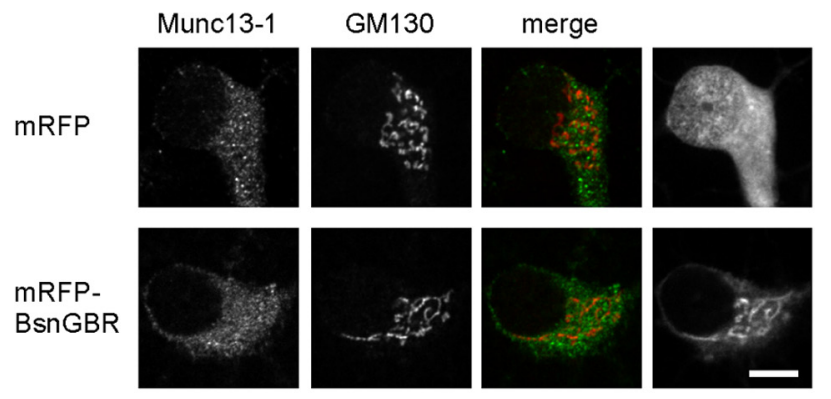

B

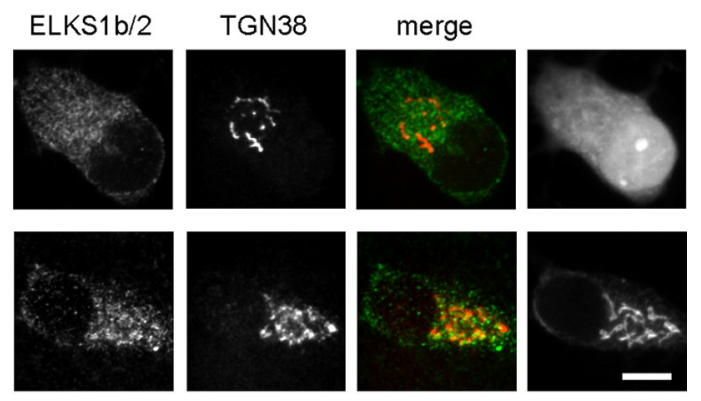

D

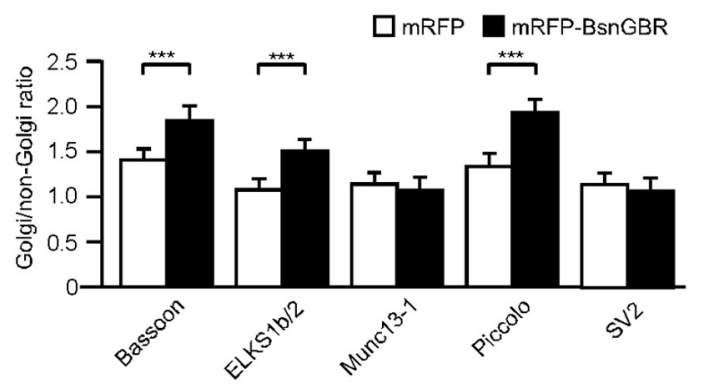

E control

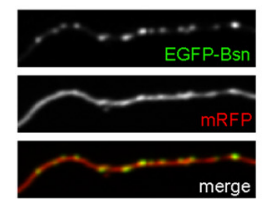

G control
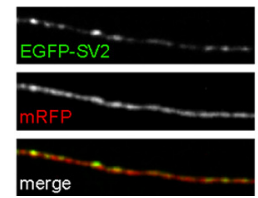

BsnGBR

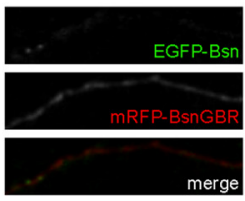

BsnGBR

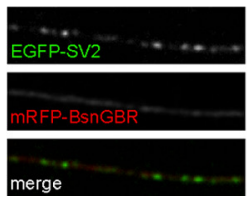

Arf-Q71।

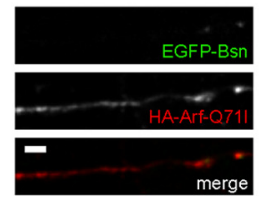

Arf-Q71।

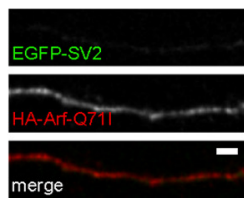

F control

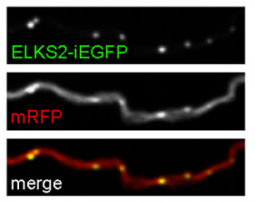

H control

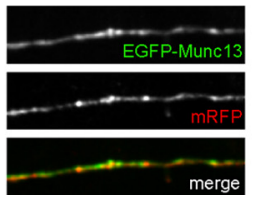

BsnGBR

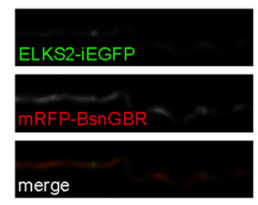

BsnGBR

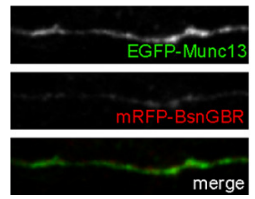

Arf-Q71I

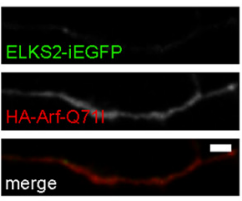

Arf-Q71।

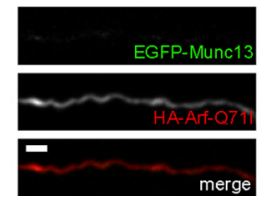

Figure 4. Distribution of Bassoon, ELKS1b/2, Munc13-1, Piccolo, and SV2 in neurons expressing mRFP or the Golgi binding region of Bassoon (mRFP-BsnGBR). A-C, Confocal images of hippocampal neurons 9 DIV immunostained with antibodies against the Golgi markers TGN38 $(\boldsymbol{A}, \boldsymbol{B})$ or GM130 (C) as well as Bassoon $(\boldsymbol{A})$, ELKS1b/2 (B), or Munc13-1 ( $\boldsymbol{C}$, respectively, after expressing $\operatorname{mRFP}(\boldsymbol{A}-\boldsymbol{C}$, first row) or mRFP-BsnGBR ( $\boldsymbol{A}-\boldsymbol{C}$, second row) for $14 \mathrm{~h}$. Scale bars $(\boldsymbol{A}-\boldsymbol{C}), 5 \mu \mathrm{m}$. D, Quantification of $\boldsymbol{A}-\boldsymbol{C}$ and respective experiments with antibodies against Piccolo and SV2. Data are represented as mean $\pm S D$, ${ }^{* * *} p<0.005, N=30$ cells from three separate experiments for each quantification. $\boldsymbol{E}-\boldsymbol{H}$, Axonal targeting of EGFP fusion proteins of Bassoon $(\boldsymbol{E})$, ELKS2 $(\boldsymbol{F})$, SV2 (G), and Munc13-1 $(\boldsymbol{H})$ in neurons coexpressing mRFP (control, first columns), mRFP-BsnGBR (second columns), or Arf-Q71I-HA (third columns). Scale bars (E- $\boldsymbol{H}), 1 \mu \mathrm{m}$.

Bassoon of $43.3 \pm 10.0 \%$ and $34.1 \pm 6.4 \%$ was significantly less (Fig. 5E).

To monitor the active transport of CAZ-associated membranes, we conducted live cell imaging experiments with recombinant EGFP-tagged CAZ proteins expressed in cultured hippocampal neurons. Initially, we verified that EGFP-fusion proteins of Bassoon, ELKS2, Munc13-1, and SV2 were appropriately localized within presynaptic boutons of synapses formed between neurons after 14 DIV (Bresler et al., 2004; Fisher-Lavie et al. 2011; data not shown). These data indicate that the EGFP tags do not interfere with synaptic delivery. We then examined the dynamics of each of the EGFP-tagged proteins within axons. Time-lapse video microscopy revealed that individual EGFPpositive Bassoon, ELKS2, Munc13-1, and SV2 puncta were highly dynamic within the axons of immature DIV6 neurons (Fig. 6A$D)$. Note that EGFP-tagged Munc13-1 is localized in clusters as well as diffused throughout the neuron, consistent with the detection of endogenous Munc13-1 in both soluble $\left(\mathrm{S}_{100}\right)$ as well as membrane bound $\left(\mathrm{P}_{100}\right)$ fractions in $\mathrm{P} 2$ lysates (Fig. $\left.2 A\right)$. The average velocities of mobile EGFP-positive puncta for all four proteins are in the range of other vesicular cargos in neurons (Hirokawa et al., 2009) and do not differ significantly from each other (Fig. 6 E). However, while the movement patterns of EGFPBassoon and ELKS2-iEGFP are quite similar, they differ from those of EGFP-tagged Munc13-1 and SV2 (Fig. 6E,F). Specifically, EGFP-tagged Bassoon and ELKS2 move in a more saltatory pattern, with breaks and directional changes of the movement as indicated by the average movement length of single movement events and shown in the kymographs (Fig. 6E,F). On the other hand, EGFP-tagged Munc13-1 and SV2 demonstrate a higher processivity, resulting in longer movement events with fewer breaks in between (Fig. 6E, F).

Finally, we performed time-lapse video microscopy of cultured primary hippocampal neurons (6 DIV) coexpressing mRFP-tagged Bassoon and EGFP-tagged ELKS2 or Munc13-1. Whereas mRFP-Bassoon and ELKS2-iEGFP fluorescent puncta 
move in close association with each other, comovement of mRFP-Bassoon and EGFP-Munc13-1 was not readily detectable (Fig. 6G,H). These data are consistent with Bassoon and ELKS2 sharing a common transport vesicle distinct from those used by Munc13-1 or SV2.

\section{Bassoon and Piccolo are necessary for the forward trafficking of ELKS2 out of the Golgi}

Our experiments with the Golgi binding region of Bassoon (mRFP-BsnGBR) suggest that Bassoon and possibly also Piccolo are critically involved in the de novo formation of gPTVs on Golgi membranes. To test this hypothesis, we employed short hairpin RNAs (shRNA) to eliminate the expression of Bassoon and/or Piccolo in cultured hippocampal neurons as described previously (Leal-Ortiz et al., 2008). As ELKS2 appears to be a reliable marker for de novo gPTVs formation on Golgi membranes (Figs. 4-6), we used its accumulation at the TGN as a measure of whether gPTVs formed in the absence of Bassoon and/or Piccolo. Four different combinations of shRNAs were used in these experiments. Initially, neurons were transfected with plasmids expressing EGFP and a shRNA against Bassoon (Bsn16) or Piccolo (Pclo28) (Leal-Ortiz et al., 2008) on the day of plating and then fixed and stained with antibodies against TGN38 and an ELKS2-specific antibody at 10 DIV. To achieve double knockdown, DKD, of Piccolo and Bassoon, we created a third vector expressing both Bsn 16 and Pclo28 (pEGFP/ DKD). As a control, a fourth vector was created expressing scrambled shRNA sequences for both Bassoon and Piccolo (DKDsc). The effectiveness of these constructs was assessed by immunoblot analysis of cellular lysates of neurons infected with a lentiviral version of these vectors. Here, DKD but not DKDsc leads to a $\geq 90 \%$ reduction of Bassoon and Piccolo expression (Fig. 7A).

Compared to control cells expressing DKDsc, we observed no change in the Golgi levels of ELKS2 immunoreactivity in neurons lacking Piccolo (Pclo28) or Bassoon (Bsn16) (Fig. 7 B, D). In contrast, downregulating the expression of Piccolo and Bassoon (DKD) resulted in the accumulation of ELKS2 at the Golgi of DIV10 neurons but not Munc13-1 (Fig. 7C,D). As a control, we performed a rescue experiment by expressing an mRFP-tagged shRNA resistant full-length Bassoon (mRFP-Bsn ${ }^{*}$ ) in DKD neurons. In DIV10 neurons cotransfected by electroporation at 0 DIV with mRFP-Bsn* and EGFP/DKD, ELKS2 no longer accumulated at the Golgi (Fig. $7 B, D$ ). Together, these data indicate that the presence of either Piccolo or Bassoon is necessary for ELKS2 to leave the Golgi in association with vesicles, but not for the recruitment of ELKS2 to Golgi membranes.

\section{The Bassoon core domain is sufficient to form gPTVs and reach synaptic sites}

To determine which parts of Bassoon are sufficient for gPTV formation, we expressed subregions of Bassoon tagged with mRFP (Fig. 8A) in DKD-expressing neurons. Previous studies showed that the central core domain of Bassoon (BsnCD, residues 1692-3263) was sufficient to be targeted to the synapse in wild-type neurons (Dresbach et al., 2003). As discussed above, this region of Bassoon contains among others the CC2 homo/ hetero-oligomerization domain (Fig. 3), the ELKS-interacting
CC3 domain (Deguchi-Tawarada et al., 2004; Jose et al., 2008), as well as a binding site for CtBP1, a protein involved in the fission of vesicular membranes budding from Golgi membrane (tom Dieck et al., 2005). The other three constructs (BsnGBR, BsnCtBP1-CC2, and BsnCC2-CC3) used in the following experiments harbor just subsets of these three domains (see Fig. $8 \mathrm{~A}$ ).

Fusion proteins of these four constructs tagged with mRFP and expressed in DKD neurons 10 DIV localized at the Golgi (Fig. $8 B-E)$. However, only mRFP-BsnCD and mRFP-BsnCtBP1$\mathrm{CC} 2$ were observed to be transported out of the Golgi and into the axons of neurons coexpressing DKD/EGFP (Fig. $8 F, H$ ). Both mRFP-BsnGBR and mRFP-BsnCC2-CC3 were selectively localized at the Golgi and were absent from proximal axon segments in DKD neurons (Fig. 8G,I), similar to wild-type neurons (Fig. 4; data not shown). Rescue of the Golgi accumulation of ELKS2 in DKD-expressing neurons could be achieved by expressing BsnCD but not BsnCtBP1-CC2, which lacks the CC3 domain necessary for Bassoon-ELKS interaction (data not shown). BsnGBR and BsnCC2-CC3, on the other hand, caused the Golgi accumulation of endogenous Bassoon and ELKS in wild-type neurons (Fig. 4; data not shown), indicating that they can act as a dominant negative to disrupt the forward trafficking of gPTVassociated AZ protein complexes.

Finally, we examined whether BsnCD and/or BsnCtBP1-CC2 localize at presynaptic sites of DKD-expressing neurons. Axonal clusters of both constructs colocalize with the postsynaptic marker Homer, confirming their synaptic localization (Fig. 8 $J, K)$. Together, these data suggest that BsnCD is sufficient to facilitate formation of a Golgi-derived vesicle containing ELKS2. Moreover, they reveal that the efficient forward trafficking of Piccolo, Bassoon, and ELKS2 via Golgi-derived vesicular intermediates requires a combination of four elements: (1) the $\mathrm{N}$ terminus of BsnCD, which contains a CtBP1 binding site, (2) a Piccolo/Bassoon homo-/hetero-oligomerization domain (CC2/ BsnGBR), (3) a binding site of for ELKS2 (CC3), as well as (4) a so far unknown docking sites for Piccolo, Bassoon, and ELKS2 on Golgi membranes.

\section{Discussion}

The CAZ is thought to spatially control SV docking and cycling within presynaptic boutons (Garner et al., 2000). Although previous studies have shown that CAZ proteins associated with precursor vesicles (called PTVs) can be quantally delivered to nascent synaptic sites (Shapira et al., 2003), the cellular/molecular mechanisms guiding the assembly of these vesicles remain 
A

EGFP-Bassoon
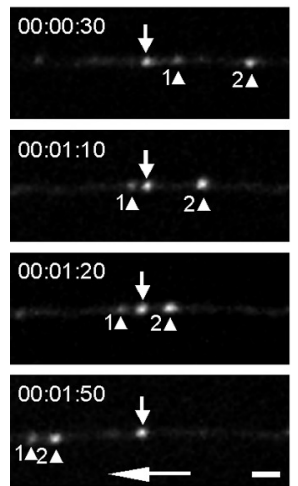

E
B

ELKS2-iEGFP
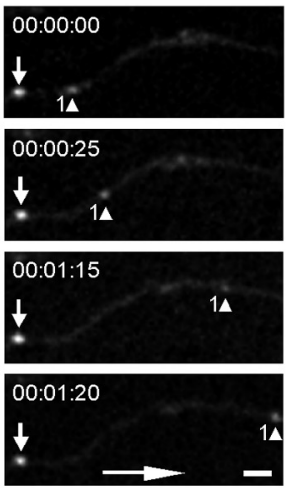

Average velocity $(\mu \mathrm{m} / \mathrm{sec})$
C

EGFP-Munc13-1
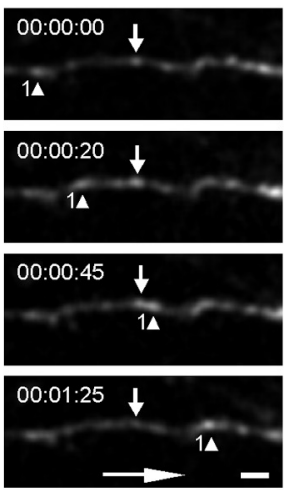

D

EGFP-SV2
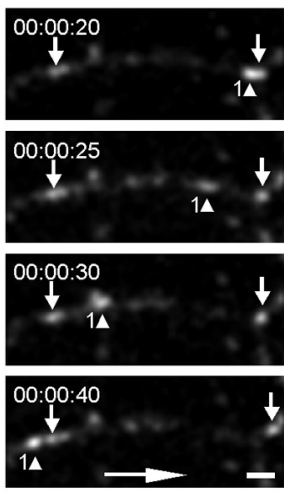

\begin{tabular}{lcc} 
& Average velocity $(\mu \mathrm{m} / \mathrm{sec})$ & Average movement length $(\mu \mathrm{m})$ \\
\hline EGFP-Bsn & $0.48 \pm 0.03$ & $2.13 \pm 0.21$ \\
\hline ELKS2-iEGFP & $0.47 \pm 0.02$ & $1.98 \pm 0.17$ \\
\hline EGFP-Munc13 & $0.56 \pm 0.03$ & $6.68 \pm 1.41$ \\
\hline EGFP-SV2 & $0.69 \pm 0.03$ & $9.54 \pm 1.25$
\end{tabular}

$\mathrm{F}$
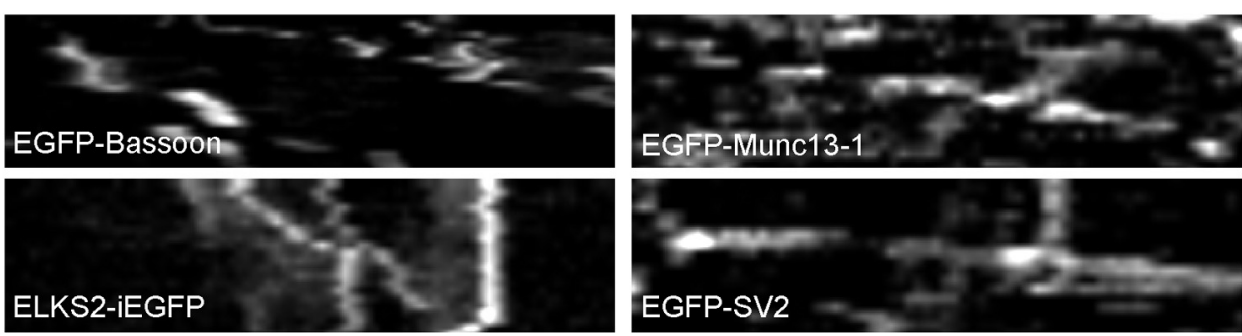

G

mRFP-Bsn
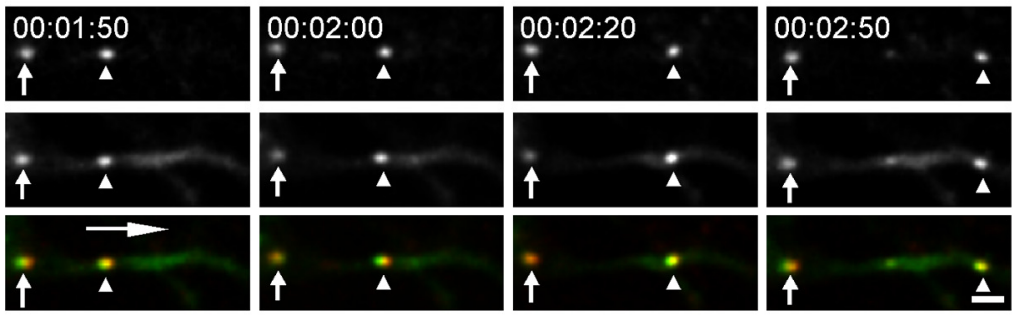

$\mathrm{H}$

mRFP-Bsn

EGFP-Munc13-1
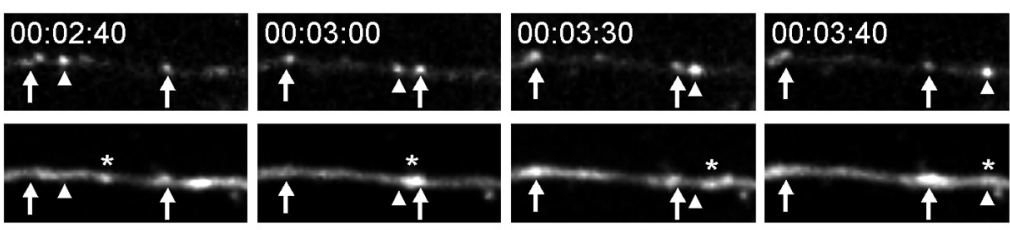

merge
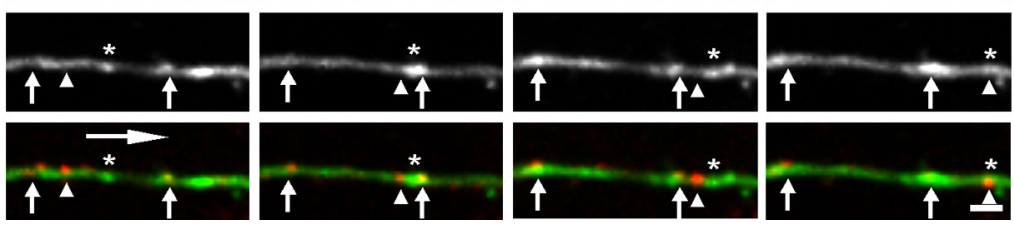

Figure 6. Active zone proteins are subject to active transport in axons of cultured hippocampal neurons. $\boldsymbol{A}-\boldsymbol{D}$, Confocal time-lapse live cell images of hippocampal neurons expressing EGFP fusion proteins of Bassoon (A), ELKS2 (B), Munc13-1 ( $)$, and SV2 (D). Vertical arrows indicate immobile signals, and arrowheads indicate mobile signals. Horizontal arrows indicate the movement direction of arrowhead-marked puncta. $\boldsymbol{E}$, Movement parameters include average velocity $(\mu \mathrm{m} / \mathrm{s})$ and average single movement length $(\mu \mathrm{m})$. Data are represented as mean $\pm S E M, N=30 \mathrm{moving}$ puncta for each construct. Scale bars $(\boldsymbol{A}-\boldsymbol{D}), 1 \mu \mathrm{m}$. $\boldsymbol{F}$, Kymographs of live cell imaging $\boldsymbol{A}-\boldsymbol{D}$ for a $50-\mu \mathrm{m}$-long portion of the axon over the course of $2.5 \mathrm{~min}$. $\boldsymbol{G}, \boldsymbol{H}$, Time-lapse live cell images of hippocampal neurons coexpressing mRFP-Bsn and ELKS2-iEGFP (G) or EGFP-Munc13-1 $(\boldsymbol{H})$, respectively. Vertical arrows indicate immobile EGFP-Bassoon puncta, and arrowheads indicate mobile EGFP-Bassoon puncta. Stars indicate mobile Munc13-1 puncta moving independently from EGFP-Bassoon. Horizontal arrows indicate the movement direction of arrowhead- and star-marked puncta. Scale bars $(\boldsymbol{F}, \boldsymbol{G}), 1 \mu \mathrm{m}$. 
A
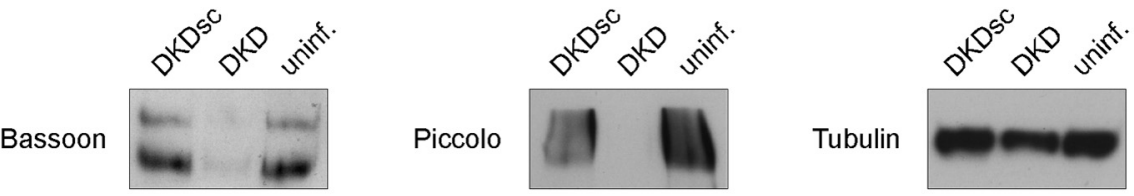

B
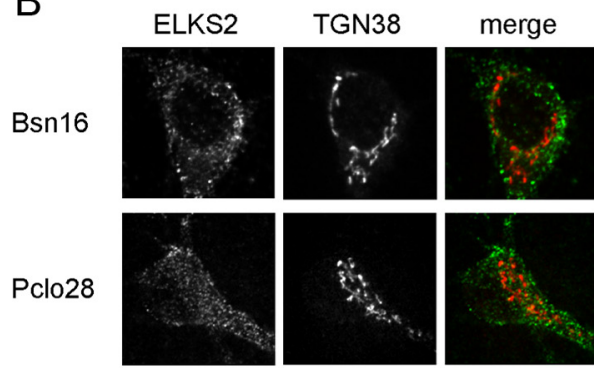

DKD
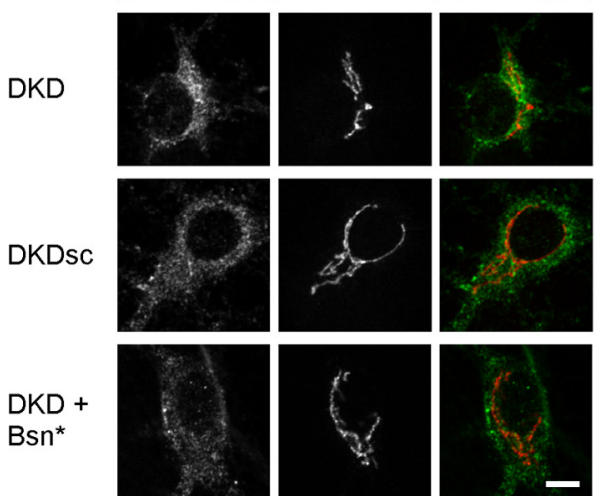

C

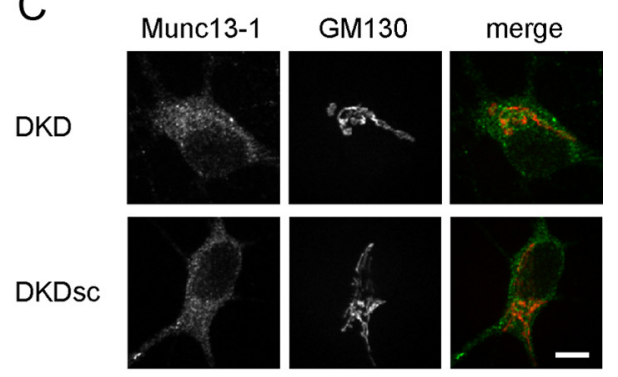

D

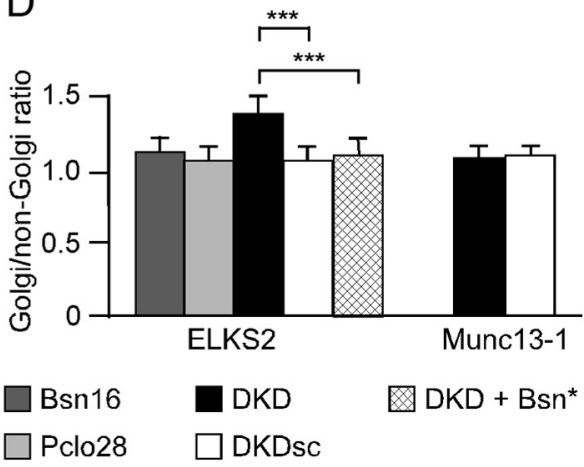

Figure 7. Effect of Bassoon and Piccolo single or double knockdown on the somatic localization of endogenous ELKS2 and Munc13-1. $\boldsymbol{A}$, Extracts of DKD-expressing neurons were analyzed by Western blotting for the distribution of Piccolo and Bassoon. Tubulin served as a loading control. uninf., Uninfected. $\boldsymbol{B}$, Confocal images of hippocampal neurons expressing shRNA constructs to knock down the expression of Bassoon (Bsn16, first row), Piccolo (Pclo28, second row), or both Bassoon and Piccolo (DKD, third row) that were immunostained with antibodies against ELKS2 and TGN38. Expression of scrambled sequences of Bsn16 and Pcl028 served as a control (DKDsc, fourth row). Expression of mRFP-tagged shRNA insensitive full-length Bassoon in DKD-expressing neurons prevented the Golgi accumulation of ELKS2 (DKD + Bsn*, fifth row). C, Immunostaining of hippocampal neurons expressing a shRNA construct to knockdown the expression of both Bassoon and Piccolo (DKD, first row) with antibodies against Munc13-1 and GM130. Expressing a scrambled sequence served as a control (DKDsc, second row). Scale bars ( $\boldsymbol{B}, \boldsymbol{C}), 5 \mu \mathrm{m}$. $\boldsymbol{D}, \mathrm{Quantification}$ of $\boldsymbol{A}$ and B. Data are represented as mean $\pm S D,{ }^{* * *} p<0.005, N=30$ cells for three separate experiments for each quantification.

poorly understood. Here, we show that CAZ protein delivery to nascent synapses likely occurs in a multistep process, as initially not all take the same routes out of the soma. For example, while three CAZ proteins, Piccolo, Bassoon, and ELKS2, were found to share a common Golgi-derived transport vesicle (gPTV), Munc13-1 transits out of the soma via a distinct Golgi-derived vesicle (Fig. 9). Surprisingly, RIM1 $\alpha$ was found to associate with transport vesicles in a post-Golgi compartment. These results suggest that gPTVs gather additional CAZ proteins (RIM1 $\alpha$ and possible Munc13) in a post-Golgi step forming a "mature" PTV, mPTV, that may ultimately participate in synapse formation (Shapira et al., 2003). Intriguingly, we also found that Piccolo and/or Bassoon are necessary and sufficient for the formation of gPTVs.

\section{Different kinds of active zone precursor vesicles contribute} to synaptogenesis

Though Piccolo and Bassoon are primarily associated with $80 \mathrm{~nm}$ dense core vesicles in immature neurons (Zhai et al., 2001; Shapira et al., 2003), they appear also to associate with morphologically distinct subtypes of vesicular membranes (Tao-Cheng, 2007). Although it is unclear which arise via de novo synthesized or membrane recycling, these data suggest that the biogenesis, delivery, and reuse of CAZ proteins are more complex than initially imagined and require more in depth studies on individual steps. In examining the first biogenic steps, we observed that the forward trafficking of Bassoon, Piccolo, ELKS2, and Munc13-1 into axons appears to start at the Golgi, as blocking the budding of vesicles from the TGN caused their accumulation at Golgi membranes. Surprisingly, RIM $1 \alpha$ did not accumulate at the Golgi, suggesting that it is recruited to vesicular intermediates in a post-Golgi step.

Intriguingly, Munc13-1 was found to use a Golgi-derived vesicle distinct from gPTVs. This is supported by our data showing that disrupting formation of gPTVs at the Golgi does not affect forward trafficking of Munc13-1. Moreover, processivity of EGFP-Munc13-1 is significantly higher than either EGFPBassoon- or ELKS2-iEGFP-associated movement (Fig. 6). This conclusion is consistent with studies demonstrating a dominantnegative construct that blocks binding of dynein light chain to full-length Bassoon-disrupted transport of Bassoon but not Munc13-1 (Fejtova et al., 2009).

Studies by Ahmari and colleagues (2000) have found clusters of vesicular and tubulovesicular membranes present at nascent synapses, implying that multiple vesicular intermediates may contribute to the rapid assembly of these junctions (Ahmari et al., 2000; Sabo and McAllister, 2003), which is consistent with the presence of different precursor vesicles. At present it is unclear how many are devoted to the formation of the AZ. Importantly, previous studies have shown that Munc13-1 and RIM1 $\alpha$ coim- 
A

A
Bassoon
BsnCD (aa 1692-3263)
BsnGBR (aa 2088-2563)
BsnCtBP1-CC2 (aa 1692-2563)
BsnCC2-CC3 (aa 2088-3263)

B
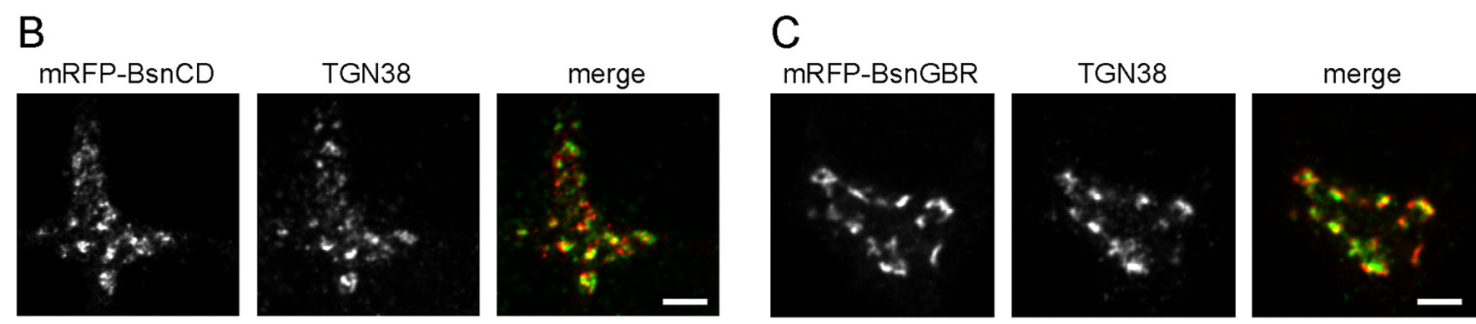

D mRFP-
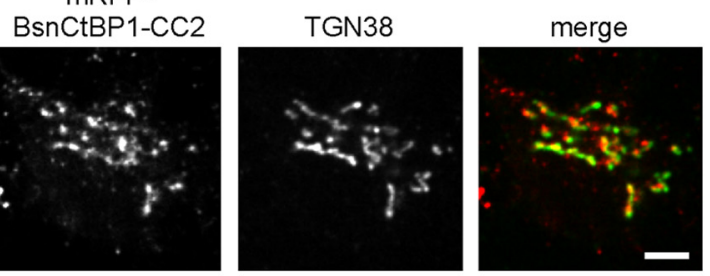

E mRFP-
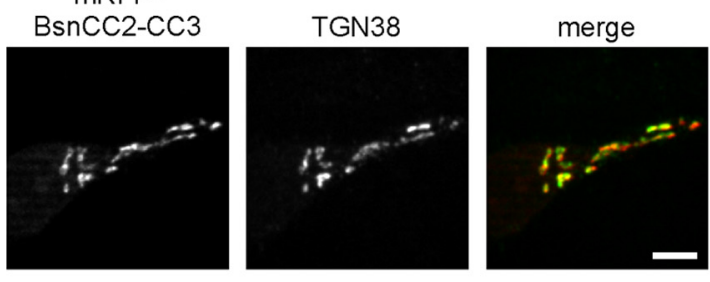

$\mathrm{F}$
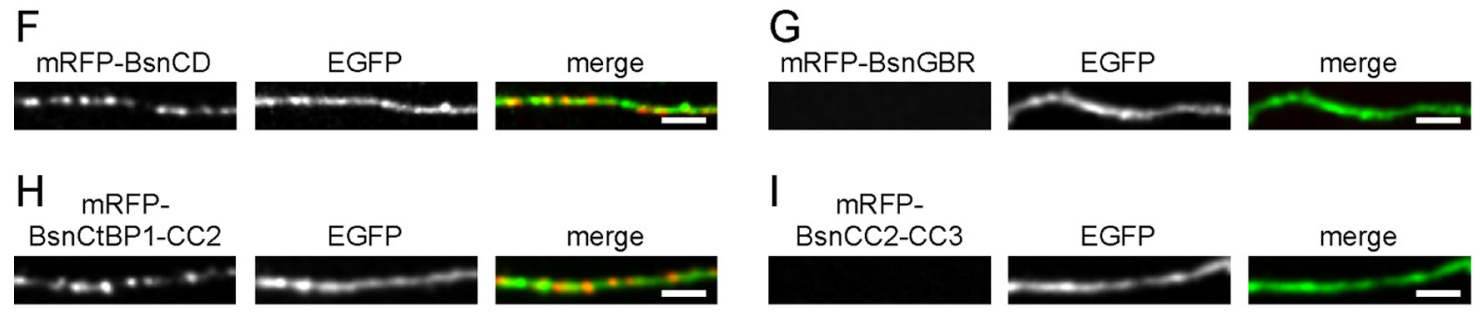

merge
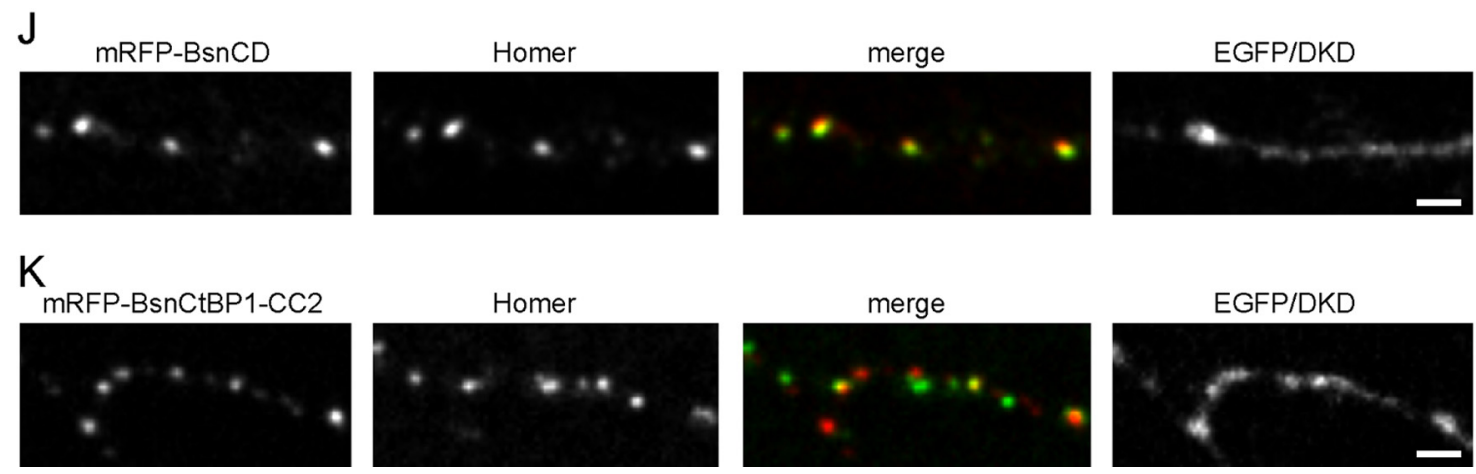

Figure 8. Localization of different fragments of Bassoon in DKD-expressing neurons. $A$, Scheme of the different fragments used in this analysis and overview of their behavior in COS-7 cells (cocluster with BsnGBR) and DKD-expressing neurons (Golgi localization, Synaptic localization). Zn, Zinc finger domains; cc, coiled-coil regions; black line, CtBP1 binding site of Bassoon. $\boldsymbol{B}-\boldsymbol{E}$, Confocal images of somata of DKD neurons expressing mRFP fusion proteins of BsnCD $(\boldsymbol{B}), B \operatorname{Bn} \mathrm{GBR}(\boldsymbol{C}), \mathrm{BsnCtBP}-\mathrm{CC}(\boldsymbol{D})$, or BsnCC2-CC3 (E), respectively, and stained with antibodies against the trans-Golgi network protein TGN38. $\boldsymbol{F}-\boldsymbol{I}$, Axons of DKD/EGFP neurons expressing mRFP fusion proteins of BsnCD $(\boldsymbol{F})$, BsnGBR $(\boldsymbol{G})$, BsnCtBP1-CC2 $(\boldsymbol{H})$, or BsnCC2-CC3 $(\boldsymbol{I})$, respectively. J, $\boldsymbol{K}$, DKD neurons expressing mRFP-BsnCD $(\boldsymbol{J})$ or mRFP-BsnCtBP1bs-CC2 $(\boldsymbol{K})$ and stained with antibodies against the postsynaptic protein Homer. Scale bars $(\boldsymbol{B}-\boldsymbol{K}), 2 \mu \mathrm{m}$.

munoprecipitate with Bassoon and Piccolo from light membrane fractions (Ohtsuka et al., 2002; Shapira et al., 2003) and associate with nonsynaptic axonal packets of Bassoon and Piccolo (Shapira et al., 2003) in distal axons and growth cones, suggesting that they eventual coalesce into a common AZ precursor vesicle (Shapira et al., 2003). Mechanistically, it is conceivable that gPTVs and vesicles carrying Munc13-1 may undergo further maturation steps that bring them together in a post-Golgi compartment to create fully mature AZ precursor vesicles (e.g., mPTVs) (Fig. 9). A similar model has been invoked to explain the observation that SV 


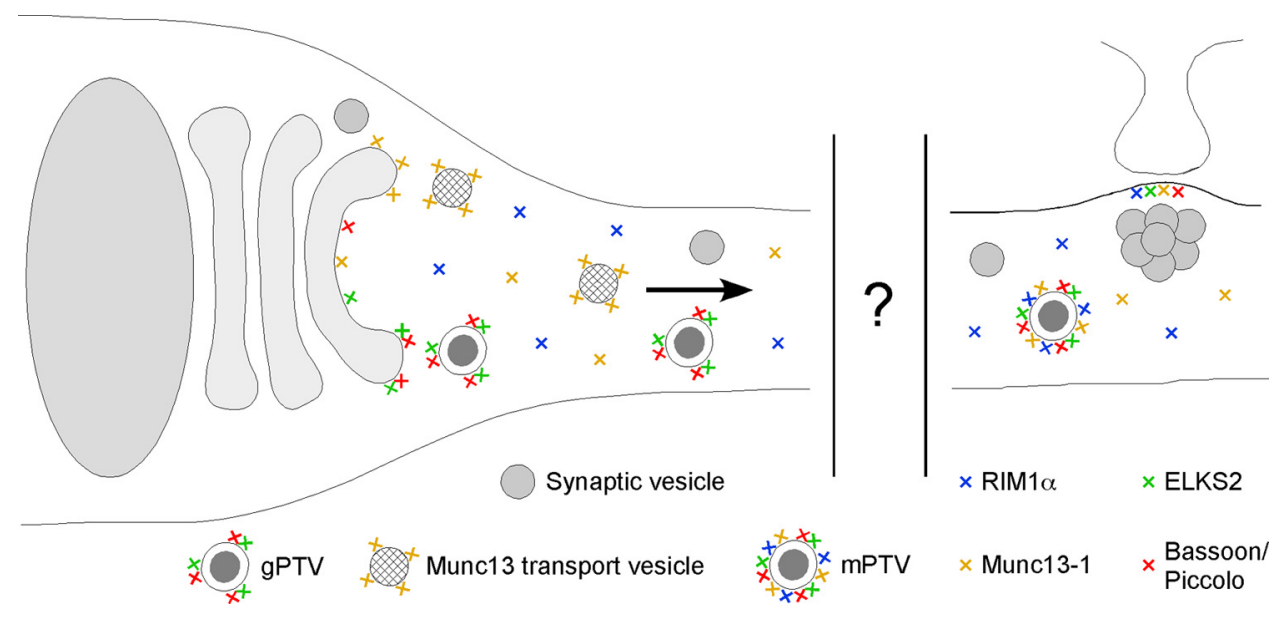

Figure 9. Schematic of active zone precursor biogenesis. Bassoon/Piccolo, ELKS2, and Munc13-1 have distinct binding sites at the Golgi, whereas RIM1 $\alpha$ does not associate with Golgi membranes. Bassoon/Piccolo and ELKS2 are sorted onto the same Golgi-derived transport vesicle, gPTV, while Munc13-1 is sorted onto its own Golgi-derived vesicle (Munc13 transport vesicle). During transport of these vesicles through the axon, they undergo so far unknown maturation steps (?) resulting in "mature" PTVs (mPTV) containing a sufficient set of active zone proteins to form presynaptic boutons. Synaptic vesicles, which also undergo additional post-Golgi maturation steps, are distinct from these active zone precursor vesicles.

proteins, e.g., Synaptophysin and SV2, leave the Golgi on distinct vesicles and form mature SVs at or near presynaptic sites after recycling with the plasma membrane (Hannah et al., 1999; Matteoli et al., 1992, 2004). Alternatively, conversion from gPTV to "mPTVs" might only involve subtle changes in composition e.g., subsequent recruitment of other CAZ proteins like Munc13-1 and RIM1 $\alpha$ from soluble pools. This scenario would also be consistent with earlier reports that CAZ proteins can be coimmunoprecipitated from light-membrane fractions and colocalize to discrete, dynamic, nonsynaptic structures in distal axons and growth cones (Ohtsuka et al., 2002; Shapira et al., 2003). It should be noted that although the majority of Munc13-1 and RIM1 $\alpha$ do not appear to be targeted into the axon on gPTVs, small numbers of molecules could still be associated with gPTVs at levels below the detection limit of the experiments used in this study.

Intriguingly, significant amounts of both Munc13-1 and RIM $1 \alpha$ exist in soluble pools during early development (Fig. 2). As these proteins are subject to active transport as well, both active and passive mechanisms might contribute to their recruitment into nascent and mature synapses. A similar scenario has been reported for PSD-95, which exhibits active transport but can also be recruited into the PSD from soluble pools (Bresler et al., 2001; Prange and Murphy, 2001). Conceptually, the dynamic association of subset of CAZ with precursor vesicles could enable neurons to temporally and spatially control their maturation and, thus, when and where nascent synapses are formed; e.g., not before axons reach appropriate receptive fields.

\section{Bassoon and Piccolo define their own transport vesicle}

Downregulation of Bassoon and Piccolo by shRNA leads to the accumulation of ELKS2 at Golgi membranes, an observation mirrored by overexpression of the Golgi-binding region of Bassoon (BsnGBD). The specificity of this effect, e.g., Golgi accumulation of ELKS2 but not Munc13-1 or SV2, implies that it is due to selective loss of Piccolo and Bassoon and not to general disruption of membrane trafficking. This conclusion is further supported by our ability to restore the forward trafficking of ELKS2 from the Golgi in cells coexpressing shRNA-insensitive fulllength Bassoon. Importantly, knockdown of Piccolo or Bassoon alone was insufficient to cause Golgi accumulation of ELKS2. We therefore conclude that the presence of either Bassoon or Piccolo is necessary for the formation of gPTVs. However, with the exception of ELKS2, we cannot exclude the possibility that proteins usually transported on gPTVs leave the Golgi on different kinds of vesicles under Piccolo/Bassoon knockdown conditions.

An open question raised by these experiments is how Piccolo and/or Bassoon contribute to the formation of gPTVs. As large scaffold proteins, it is conceivable that Bassoon and Piccolo use their multidomain structures and ability to form complexes with each other to create a structural lattice that facilitates formation of gPTVs and recruits other key AZ proteins. Oligomerization of Piccolo and Bassoon via direct interaction through the Golgibinding domain in these molecules potentially forms the basis for the assembly of this structure. This region not only can mediate Bassoon/Piccolo oligomerization but also appears to be an attachment site to the Golgi, as its accumulation on the Golgi occurs in the absence of Piccolo and Bassoon.

Previous studies showed that the CC2 domain in ELKS2 directly binds the CC3 domain in Piccolo and Bassoon (Ohtsuka et al., 2002; Deguchi-Tawarada et al., 2004). It is thus possible that ELKS2 uses this interaction to draw itself into newly forming gPTVs. This conclusion is also supported by the finding that the BsnCD construct, which contains Bassoon's CC3 domain, can release ELKS2 from the Golgi in DKD neurons, whereas BsnCtBP1-CC2, which lacks CC3, cannot although BsnCtBP1CC2 can leave the Golgi itself (Fig. 8). The accumulation of ELKS2 on Golgi membranes in the absence of Piccolo and Bassoon indicates that it has a binding partner on Golgi membranes other than Piccolo and Bassoon. A possible candidate is Rab6, a Golgi-associated protein that also binds ELKS2 (Grigoriev et al., 2007).

Our structure-function studies also reveal that sequences upstream of the GBR and present in BsnCD are important for forward trafficking of gPTVs from the Golgi. The N-terminal segment of BsnCD contains a binding site for carboxyl-terminal binding protein 1 (CtBP-1) (tom Dieck et al., 2005). CtBP-3, a splice variant of $\mathrm{CtBP} 1$, is part of the machinery controlling vesicle fission at the Golgi (Bonazzi et al., 2005). Interaction of CtBP-1 with Bassoon and Piccolo might thus have a similar purpose, facilitating budding and fission of gPTVs from the Golgi. This is consistent with CtBP1 loss of function experiments that 
inhibit Piccolo and Bassoon transport into axons (A. Fejtova and E. D. Gundelfinger, personal communication).

The present study provides evidence that biogenesis and transport of AZ proteins is more complex than previously thought and likely involves additional maturation steps. Our studies of the first biogenic step revealed that some CAZ proteins are sorted onto distinct Golgi-derived transport vesicles (e.g., Bassoon, Piccolo, and ELKS2 on gPTVs; Munc13-1 on a yet uncharacterized precursor vesicle) or become associated with vesicular membranes within a post-Golgi compartment (e.g., RIM1 $\alpha$ ). This specification could facilitate two aspects of synaptogenesis: the need to accumulate a large collection of presynaptic molecules at appropriate stoichiometries within a reasonable time and the necessity to tightly regulate the composition of individual presynaptic specializations. As in postsynaptic glutamate receptor transport (Jeyifous et al., 2009), we also find that synaptic scaffold proteins of the active zone use their multidomain structures to direct and define the assembly and cargo content of their own transport vesicles.

\section{References}

Ahmari SE, Buchanan J, Smith SJ (2000) Assembly of presynaptic active zones from cytoplasmic transport packets. Nat Neurosci 3:445-451.

Banker G, Goslin K (1998) Culturing nerve cells, Ed 2. Cambridge, MA: MIT.

Bonazzi M, Spanò S, Turacchio G, Cericola C, Valente C, Colanzi A, Kweon HS, Hsu VW, Polishchuck EV, Polishchuck RS, Sallese M, Pulvirenti T, Corda D, Luini A (2005) CtBP3/BARS drives membrane fission in dynamin-independent transport pathways. Nat Cell Biol 7:570-580.

Bresler T, Ramati Y, Zamorano PL, Zhai R, Garner CC, Ziv NE (2001) The dynamics of SAP90/PSD-95 recruitment to new synaptic junctions. Mol Cell Neurosci 18:149-167.

Bresler T, Shapira M, Boeckers T, Dresbach T, Futter M, Garner CC, Rosenblum K, Gundelfinger ED, Ziv NE (2004) Postsynaptic density assembly is fundamentally different from presynaptic active zone assembly. J Neurosci 24:1507-1520.

Cases-Langhoff C, Voss B, Garner AM, Appeltauer U, Takei K, Kindler S, Veh RW, De Camilli P, Gundelfinger ED, Garner CC (1996) Piccolo, a novel $420 \mathrm{kDa}$ protein associated with the presynaptic cytomatrix. Eur J Cell Biol 69:214-223.

Cotman CW, Matthews DA (1971) Synaptic plasma membranes from rat brain synaptosomes: isolation and partial characterization. Biochim Biophys Acta 249:380-394.

Dascher C, Balch WE (1994) Dominant inhibitory mutants of ARF1 block endoplasmic reticulum to Golgi transport and trigger disassembly of the Golgi apparatus. J Biol Chem 269:1437-1448.

Deguchi-Tawarada M, Inoue E, Takao-Rikitsu E, Inoue M, Ohtsuka T, Takai Y (2004) CAST2: identification and characterization of a protein structurally related to the presynaptic cytomatrix protein CAST. Genes Cells 9:15-23.

Dresbach T, Hempelmann A, Spilker C, tom Dieck S, Altrock WD, Zuschratter W, Garner CC, Gundelfinger ED (2003) Functional regions of the presynaptic cytomatrix protein bassoon: significance for synaptic targeting and cytomatrix anchoring. Mol Cell Neurosci 23:279-291.

Dresbach T, Torres V, Wittenmayer N, Altrock WD, Zamorano P, Zuschratter W, Nawrotzki R, Ziv NE, Garner CC, Gundelfinger ED (2006) Assembly of active zone precursor vesicles: obligatory trafficking of presynaptic cytomatrix proteins Bassoon and Piccolo via a trans-Golgi compartment. J Biol Chem 281:6038-6047.

Fejtova A, Davydova D, Bischof F, Lazarevic V, Altrock WD, Romorini S, Schöne C, Zuschratter W, Kreutz MR, Garner CC, Ziv NE, Gundelfinger ED (2009) Dynein light chain regulates axonal trafficking and synaptic levels of Bassoon. J Cell Biol 185:341-355.

Fisher-Lavie A, Zeidan A, Stern M, Garner CC, Ziv NE (2011) Use dependence of presynaptic tenacity. J Neurosci 31:16770-16780.

Garner CC, Kindler S, Gundelfinger ED (2000) Molecular determinants of presynaptic active zones. Curr Opin Neurobiol 10:321-327.

Grigoriev I, Splinter D, Keijzer N, Wulf PS, Demmers J, Ohtsuka T, Modesti M, Maly IV, Grosveld F, Hoogenraad CC, Akhmanova A (2007) Rab6 regulates transport and targeting of exocytotic carriers. Dev Cell 13:305-314

Hannah MJ, Schmidt AA, Huttner WB (1999) Synaptic vesicle biogenesis. Annu Rev Cell Dev Biol 15:733-798.

Hirokawa N, Noda Y, Tanaka Y, Niwa S (2009) Kinesin superfamily motor proteins and intracellular transport. Nat Rev Mol Cell Biol 10:682-696.

Huttner WB, Schiebler W, Greengard P, De Camilli P (1983) Synapsin I (protein I), a nerve terminal-specific phosphoprotein. III. Its association with synaptic vesicles studied in a highly purified synaptic vesicle preparation. J Cell Biol 96:1374-1388.

Jeyifous O, Waites CL, Specht CG, Fujisawa S, Schubert M, Lin EI, Marshall J, Aoki C, de Silva T, Montgomery JM, Garner CC, Green WN (2009) SAP97 and CASK mediate sorting of NMDA receptors through a previously unknown secretory pathway. Nat Neurosci 12:1011-1019.

Jose M, Nair DK, Altrock WD, Dresbach T, Gundelfinger ED, Zuschratter W (2008) Investigating interactions mediated by the presynaptic protein bassoon in living cells by Foerster's resonance energy transfer and fluorescence lifetime imaging microscopy. Biophys J 94:1483-1496.

Kalla S, Stern M, Basu J, Varoqueaux F, Reim K, Rosenmund C, Ziv NE, Brose N (2006) Molecular dynamics of a presynaptic active zone protein studied in Munc13-1-enhanced yellow fluorescent protein knock-in mutant mice. J Neurosci 26:13054-13066.

Langnaese K, Seidenbecher C, Wex H, Seidel B, Hartung K, Appeltauer U, Garner A, Voss B, Mueller B, Garner CC, Gundelfinger ED (1996) Protein components of a rat brain synaptic junctional protein preparation. Brain Res Mol Brain Res 42:118-122.

Leal-Ortiz S, Waites CL, Terry-Lorenzo R, Zamorano P, Gundelfinger ED, Garner CC (2008) Piccolo modulation of Synapsinla dynamics regulates synaptic vesicle exocytosis. J Cell Biol 181:831-846.

Lohse K, Helmke SM, Wood MR, Quiroga S, de la Houssaye BA, Miller VE, Negre-Aminou P, Pfenninger KH (1996) Axonal origin and purity of growth cones isolated from fetal rat brain. Brain Res Dev Brain Res 96:83-96.

Lois C, Hong EJ, Pease S, Brown EJ, Baltimore D (2002) Germline transmission and tissue-specific expression of transgenes delivered by lentiviral vectors. Science $295: 868-872$.

Maas C, Tagnaouti N, Loebrich S, Behrend B, Lappe-Siefke C, Kneussel M (2006) Neuronal cotransport of glycine receptor and the scaffold protein gephyrin. J Cell Biol 172:441-451.

Matteoli M, Takei K, Perin MS, Südhof TC, De Camilli P (1992) Exoendocytotic recycling of synaptic vesicles in developing processes of cultured hippocampal neurons. J Cell Biol 117:849-861.

Matteoli M, Coco S, Schenk U, Verderio C (2004) Vesicle turnover in developing neurons: how to build a presynaptic terminal. Trends Cell Biol 14:133-140.

Ohtsuka T, Takao-Rikitsu E, Inoue E, Inoue M, Takeuchi M, Matsubara K, Deguchi-Tawarada M, Satoh K, Morimoto K, Nakanishi H, Takai Y (2002) Cast: a novel protein of the cytomatrix at the active zone of synapses that forms a ternary complex with RIM1 and munc13-1. J Cell Biol 158:577-590.

Pfenninger KH, Ellis L, Johnson MP, Friedman LB, Somlo S (1983) Nerve growth cones isolated from fetal rat brain: subcellular fractionation and characterization. Cell 35:573-584.

Prange O, Murphy TH (2001) Modular transport of postsynaptic density-95 clusters and association with stable spine precursors during early development of cortical neurons. J Neurosci 21:9325-9333.

Sabo SL, McAllister AK (2003) Mobility and cycling of synaptic proteincontaining vesicles in axonal growth cone filopodia. Nat Neurosci 6:1264-1269.

Schoch S, Gundelfinger ED (2006) Molecular organization of the presynaptic active zone. Cell Tissue Res 326:379-391.

Schultz J, Milpetz F, Bork P, Ponting CP (1998) SMART, a simple modular architecture research tool: Identification of signaling domains. Proc Natl Acad Sci U S A 95:5857-5864.

Shapira M, Zhai RG, Dresbach T, Bresler T, Torres VI, Gundelfinger ED, Ziv NE, Garner CC (2003) Unitary assembly of presynaptic active zones from Piccolo-Bassoon transport vesicles. Neuron 38:237-252.

Sudhof TC (2004) The synaptic vesicle cycle. Annu Rev Neurosci 27:509-547.

Tao-Cheng JH (2007) Ultrastructural localization of active zone and synaptic vesicle proteins in a preassembled multi-vesicle transport aggregate. Neuroscience 150:575-584 
tom Dieck S, Sanmartí-Vila L, Langnaese K, Richter K, Kindler S, Soyke A, Wex H, Smalla KH, Kämpf U, Fränzer JT, Stumm M, Garner CC, Gundelfinger ED (1998) Bassoon, a novel zinc-finger CAG/glutaminerepeat protein selectively localized at the active zone of presynaptic nerve terminals. J Cell Biol 142:499-509.

tom Dieck S, Altrock WD, Kessels MM, Qualmann B, Regus H, Brauner D, Fejtová A, Bracko O, Gundelfinger ED, Brandstätter JH (2005) Molecular dissection of the photoreceptor ribbon synapse: physical interaction of Bassoon and RIBEYE is essential for the assembly of the ribbon complex. J Cell Biol 168:825-836.

Waites CL, Craig AM, Garner CC (2005) Mechanisms of vertebrate synaptogenesis. Annu Rev Neurosci 28:251-274.

Wang X, Hu B, Zieba A, Neumann NG, Kasper-Sonnenberg M, Honsbein A, Hultqvist G, Conze T, Witt W, Limbach C, Geitmann M, Danielson H, Kolarow R, Niemann G, Lessmann V, Kilimann MW (2009) A protein interaction node at the neurotransmitter release site: domains of Ac-
zonin/Piccolo, Bassoon, CAST, and rim converge on the N-terminal domain of Munc13-1. J Neurosci 29:12584-12596.

Yonekawa Y, Harada A, Okada Y, Funakoshi T, Kanai Y, Takei Y, Terada S, Noda T, Hirokawa N (1998) Defect in synaptic vesicle precursor transport and neuronal cell death in KIF1A motor protein-deficient mice. J Cell Biol 141:431-441.

Zhai RG, Vardinon-Friedman H, Cases-Langhoff C, Becker B, Gundelfinger ED, Ziv NE, Garner CC (2001) Assembling the presynaptic active zone: a characterization of an active one precursor vesicle. Neuron 29:131-143.

Zhai R, Olias G, Chung WJ, Lester RA, tom Dieck S, Langnaese K, Kreutz MR, Kindler S, Gundelfinger ED, Garner CC (2000) Temporal appearance of the presynaptic cytomatrix protein bassoon during synaptogenesis. Mol Cell Neurosci 15:417-428.

Ziv NE, Garner CC (2004) Cellular and molecular mechanisms of presynaptic assembly. Nat Rev Neurosci 5:385-399. 